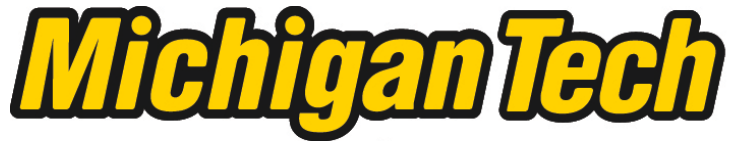 \\ Michigan Technological University Create the Future Digital Commons @ Michigan Tech
}

Dissertations, Master's Theses and Master's Reports - Open

Dissertations, Master's Theses and Master's

Reports

2012

POPLAR GENE EXPRESSION DATA ANALYSIS PIPELINES

Xiang Li

Michigan Technological University

Follow this and additional works at: https://digitalcommons.mtu.edu/etds

Part of the Computer Sciences Commons

Copyright 2012 Xiang Li

\section{Recommended Citation}

Li, Xiang, "POPLAR GENE EXPRESSION DATA ANALYSIS PIPELINES", Master's report, Michigan Technological University, 2012.

https://doi.org/10.37099/mtu.dc.etds/603

Follow this and additional works at: https://digitalcommons.mtu.edu/etds

Part of the Computer Sciences Commons 


\title{
POPLAR GENE EXPRESSION DATA ANALYSIS PIPELINES
}

\author{
By \\ Xiang Li
}

\begin{abstract}
A REPORT
Submitted in partial fulfillment of the requirements for the degree of MASTER OF SCIENCE

In Computer Science
\end{abstract}

MICHIGAN TECHNOLOGICAL UNIVERSITY

2012

(C)2012 Xiang Li 
This report has been approved in partial fulfillment of the requirements for the Degree of MASTER OF SCIENCE in Computer Science.

\title{
Department of Computer Science
}

\author{
Report Advisor: $\quad$ Dr. Hairong Wei \\ Committee Member: Dr. Laura Brown \\ Committee Member: Dr. Zhenlin Wang \\ Committee Member: Dr. Victor Bursov \\ Department Chair: Dr. Charles Wallace
}




\section{Contents}

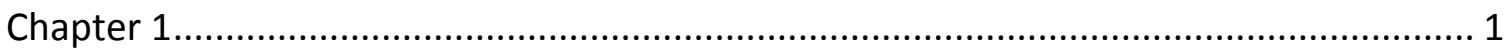

Introduction to Poplar Gene Analysis Pipeline ...................................................... 1

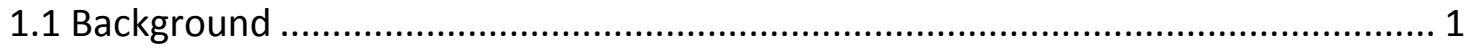

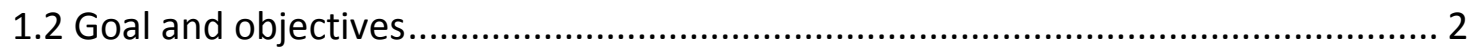

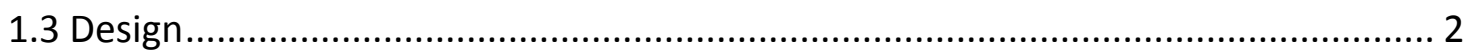

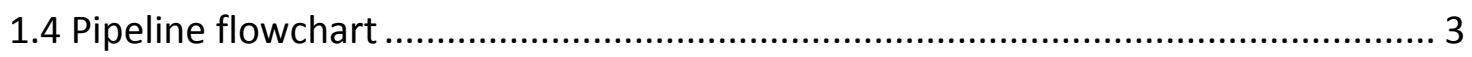

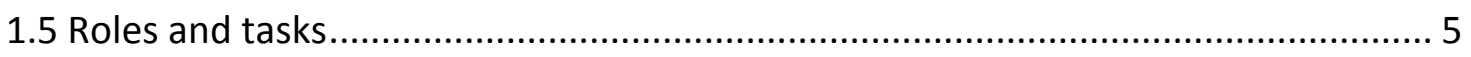

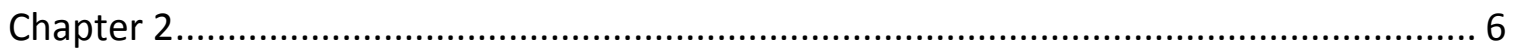

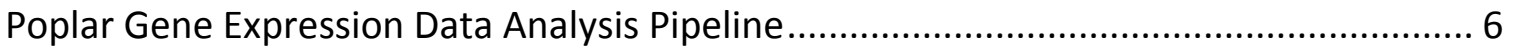

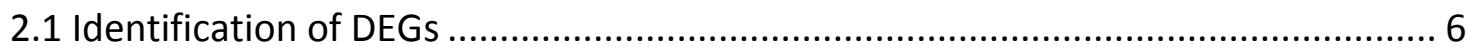

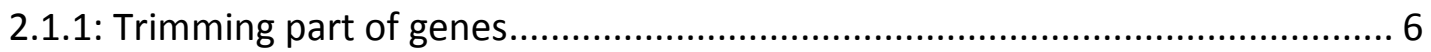

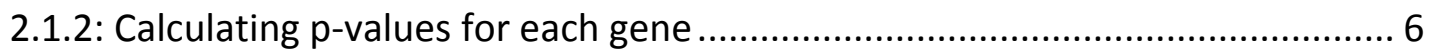

2.1.3: Trimming genes with larger $p$-values ........................................................ 8

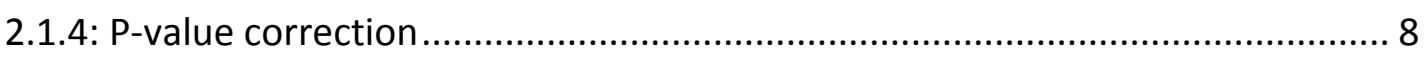

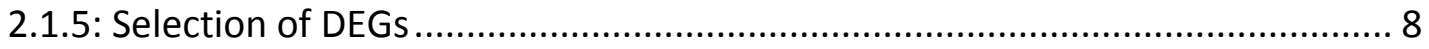

2.2 Identification of Differentially Expressed Pathways .......................................... 10

2.2.1: Calculate the fold change for each poplar gene .......................................... 11

2.2.2: Call SAM_GS program to evaluate all the pathways defined in pathway

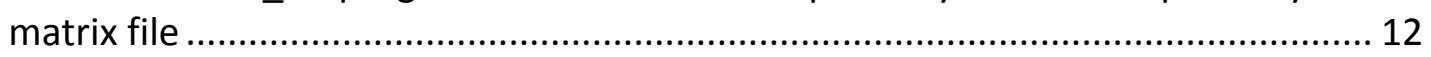

2.2.3: Calculate the average fold change for each pathway ................................ 12

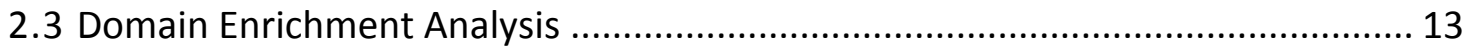

2.3.1: Calculate genome size, genome number, sample size, and sample number 13

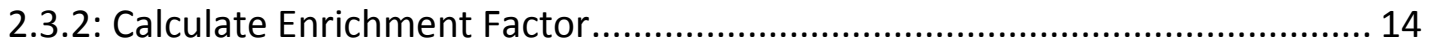

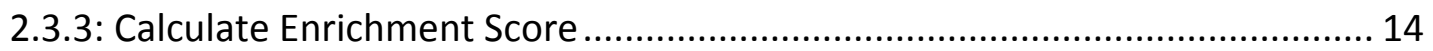

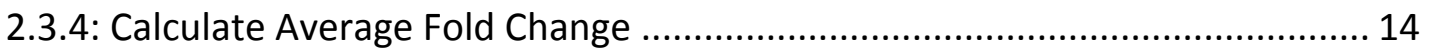

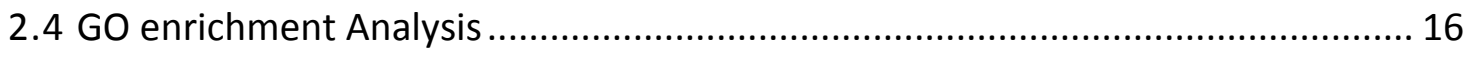

2.4.1: Generate a list of Arabidopsis genes of DEGs in each time point ................. 17

2.4.2: Call TermFinder to generate GO terms given the list of Arabidopsis genes .. 18

2.4.3: Mapping the Arabidopsis gene list of each GO back to poplar genes, and calculating the genome size, genomic gene number, sample size, sample number 18 
2.4.4: Appending the annotation of Arabidopsis genes to the result ..................... 19

2.4.5: Identification of common GO terms and Unique GO terms......................... 20

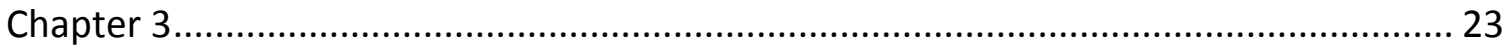

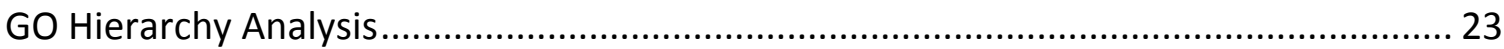

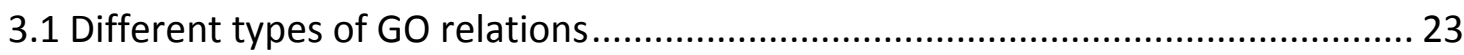

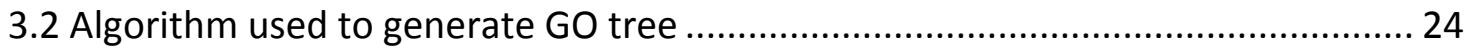

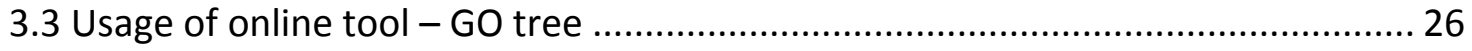

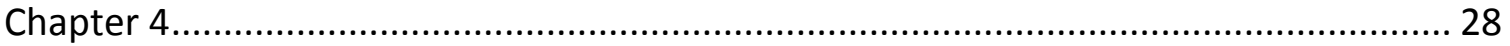

GO Hierarchy Analysis Poplar Gene Expression Data Analysis On-line Tool ................... 28

4.1 Introduction to poplar gene expression data analysis on-line tool ...................... 28

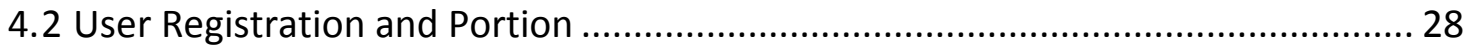

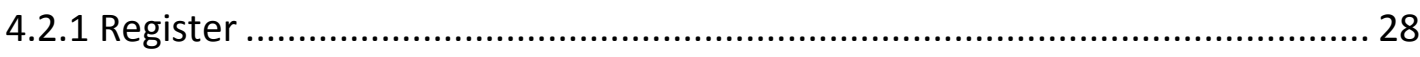

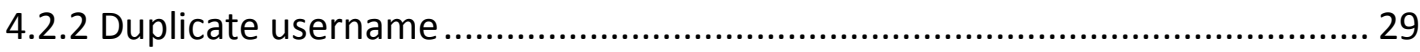

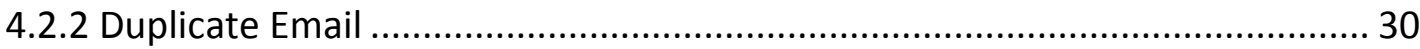

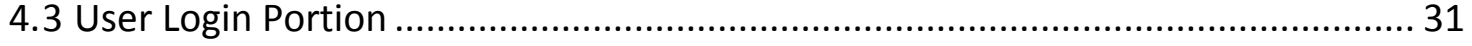

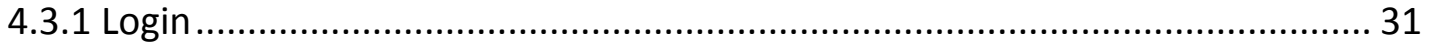

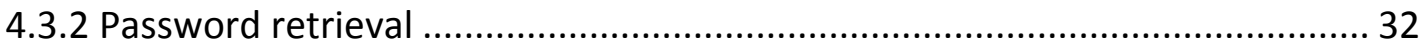

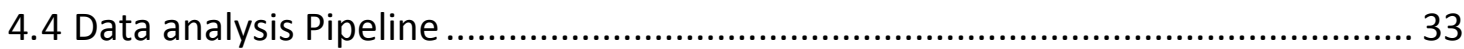

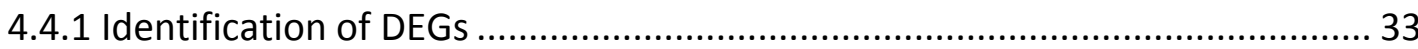

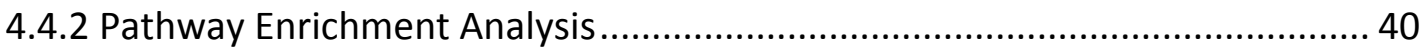

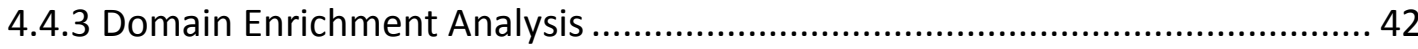

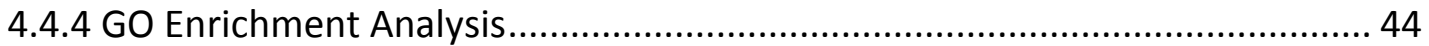

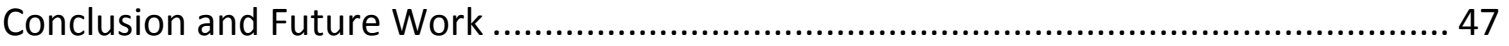

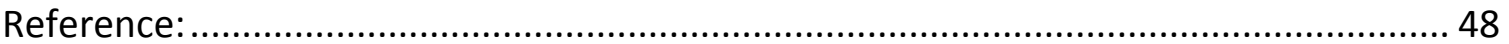




\begin{abstract}
Analyzing large-scale gene expression data is a labor-intensive and time-consuming process. To make data analysis easier, we developed a set of pipelines for rapid processing and analysis poplar gene expression data for knowledge discovery. Of all pipelines developed, differentially expressed genes (DEGs) pipeline is the one designed to identify biologically important genes that are differentially expressed in one of multiple time points for conditions. Pathway analysis pipeline was designed to identify the differentially expression metabolic pathways. Protein domain enrichment pipeline can identify the enriched protein domains present in the DEGs. Finally, Gene Ontology (GO) enrichment analysis pipeline was developed to identify the enriched GO terms in the DEGs.
\end{abstract}

Our pipeline tools can analyze both microarray gene data and highthroughput gene data. These two types of data are obtained by two different technologies. A microarray technology is to measure gene expression levels via microarray chips, a collection of microscopic DNA spots attached to a solid (glass) surface, whereas high throughput sequencing, also called as the next-generation sequencing, is a new technology to measure gene expression levels by directly sequencing mRNAs, and obtaining each mRNA's copy numbers in cells or tissues.

We also developed a web portal (http://sys.bio.mtu.edu/) to make all pipelines available to public to facilitate users to analyze their gene expression data. In addition to the analyses mentioned above, it can also perform GO hierarchy analysis, i.e. construct GO trees using a list of GO terms as an input. 


\section{Chapter 1}

\section{Introduction to Poplar Gene Analysis Pipeline}

\subsection{Background}

The DNA sequences of genes carry the codes for synthesis of functional gene product - protein. When genes are expressed, messenger RNAs (mRNAs) are transcribed by using DNA as templates, and are subsequently translated into various functional proteins. We refer to this process as gene expression. However, due to the genetic programs and various environmental conditions, not all genes are expressed at the same time and at the same levels. Different gene expression profiles can lead to the various gene dosages that define phenotypes. Different genes may participate in different biological process, and function in different tissues, and various environmental conditions. Hence analyzing gene expression data plays an essential role in getting better understanding how life functions.

DNA microarrays, or DNA chips is the technology that facilitate the parallel execution of experiments on all genomic genes simultaneously ${ }^{[1]}$. Such technology measures mRNAs in cells or tissues at any moment. In the microarray experiment, Gene-specific DNA fragments are immobilized on a glass slide. Then mRNAs extracted from control or treated tissues are labeled with

fluorescence dyes, cy 5 and cy 3 , and used to hybridize to the array under the law of base-pairing rule. After hybridization is done, we can measure the quantity of each mRNA species from the same genes by measuring the spot lit on the chip, such quantity yields raw value of gene expression. This technology is called microarray data. The data we used is yielded from Affymetrix poplar genome chips that contain 61413 gene-specific fragments for interrogating gene expression of 61413 genes

Due to the advent of new DNA sequencing technologies, high throughput sequencing technology, also called next-generation sequencing technologies are now emerged as an alternative technology to microarray for gene expression analysis ${ }^{[2]}$. The development of high throughput sequencing is driven by the high demand for low-cost sequencing. Such technique can produce thousands or millions of sequences at once ${ }^{[4]}{ }^{[5]}$ by taking advantage of parallelizing the sequencing process. High throughput sequencing is a technique that can be used directly sequencing mRNA species. Several high throughput sequencing methods are taking advantage of the power of massively-parallel sequencing to map short DNA sequence on long genome with large scale. Typically they will count the abundance of each mRNA species in cell or tissues. 
Analyzing microarray or high throughput sequencing data is tedious and very time-consuming when the data are yielded from various tissues and in a time series. To reduce the workload for biologists, we developed multiple gene expression pipelines and assembled them together to facilitate the analysis of gene expression data. We focus on the gene expression data from a bioenergy species, poplar. Poplar is not only a top-quality source of biofuel, but also the first tree species to have its entire genome sequenced. Our gene expression package as shown (http://sys.bio.mtu.edu) will not only help biologists save a lot of time but also identify important genes involved in different biological processes.

\subsection{Goal and objectives}

The goal of this project is to develop a set of tools for rapid analyzing gene expression data for model species, poplar. The tools can be used to identify DEGs, pathways, enriched protein domains and GO terms. The input data type can be either microarray data or high throughput data.

The objectives of the pipeline tools are to:

1. Analyze gene expression data for identifying DEGs and gene sets by applying different statistical methods.

2. Evaluate the results from DEG pipeline; identify the enriched protein domains and GO terms. Calculate the enrichment factors, enrichment scores and average fold changes for enriched protein domains and GO terms.

3. Perform GO hierarchy analysis of a given list of GO terms. Then construct the GO tree based on the relations among the given GO terms.

4. Implement web application of all developed pipelines with friendly user interfaces to facilitate the use of the poplar gene analysis pipeline.

My work concentrated on the development of both command line package and web application. In the web application, we allow users to upload their data obtained from their experiments. We will run multiple analyses in background and send the result links via e-mail to notify the user.

\subsection{Design}

Our pipeline line tools can take gene expression data from a time series or multiple comparisons. The tools analyze data from each time point or treatment 
independently. We use a data set of multiple time points as an example to demonstrate the usages of the developed pipelines. We have the following restrictions for gene expression files uploaded:

1. At each time point, expression file must contain control (untreated) replicates and treatment replicates. In most cases control replicates represent the information from wild type plants.

2. Due to the statistical methods we use, each time point must have at least 2 control replicates and 2 treatment replicates.

3. Data from each time point may have different number of control replicates and treatment replicates but the numbers of control replicates and treatment replicates must be consistent throughout all time points.

DEG pipeline takes the gene expression data as input and try to identify the DEGs for each time point. Here we used Rank Product method ${ }^{[3]}$ to identify the DEGs for microarray data. Pathway (or DEG set) analysis attempts to identify the DEG set by taking the gene expression data and the pathway matrix files as input. Pathway analysis is applied to gene expression data across all the time points. . The pathway matrix is a file in which each row is a gene and each column is a pathway, and a number in each intersection is either 0 or 1 . A value of one indicates this gene is in this pathway, zero otherwise. Domain and GO enrichment analyses are applied to the DEGs that were output from DEG pipeline. After getting the DEGs from each time points, we extract them separately and try to identify the corresponding protein domains and GO terms. Calculate the enrichment factors, enrichment scores as well as the average fold changes of the genes included.

\subsection{Pipeline flowchart}




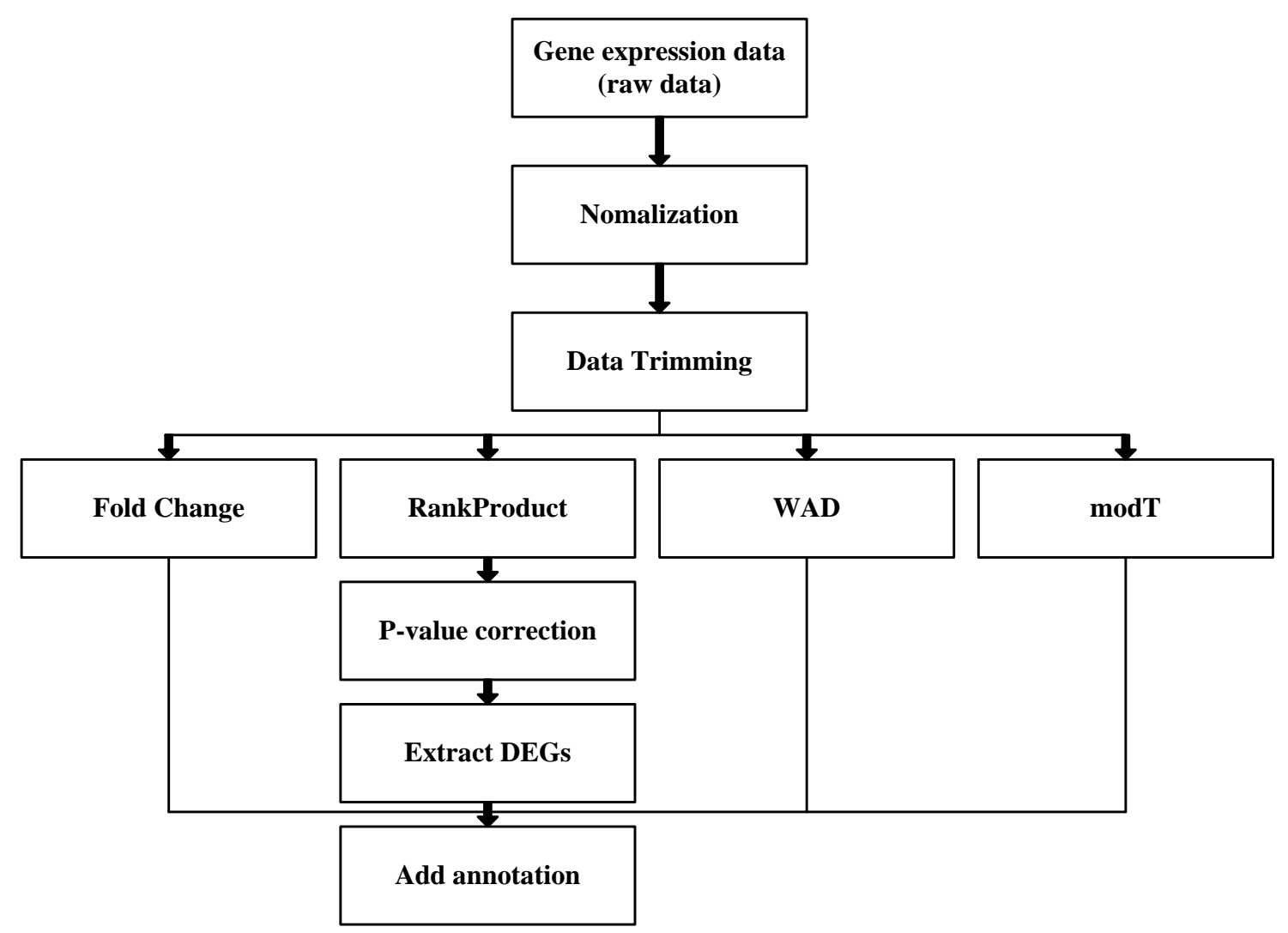

Figure 1: DEG analysis flowchart

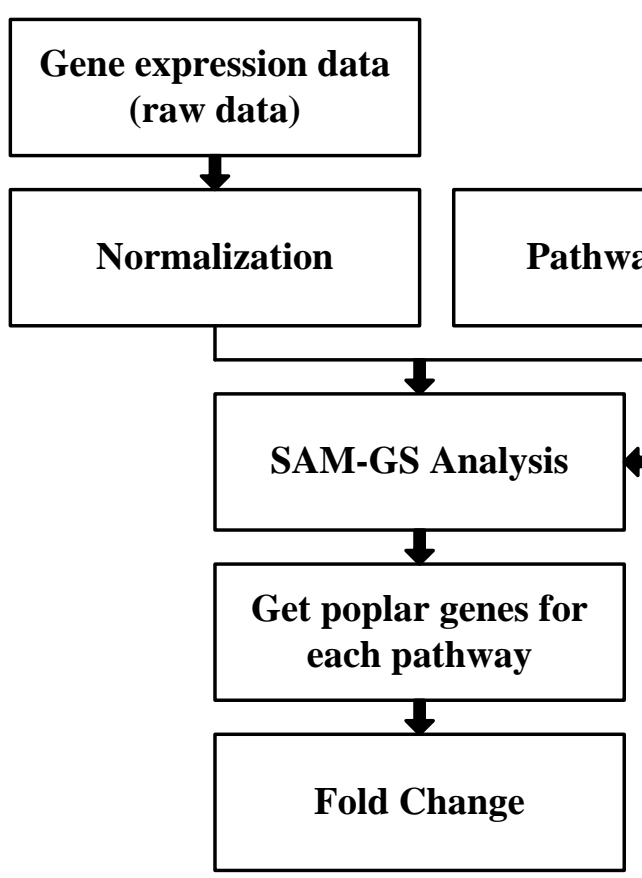

Figure 2: Pathway Analysis Flowchart 


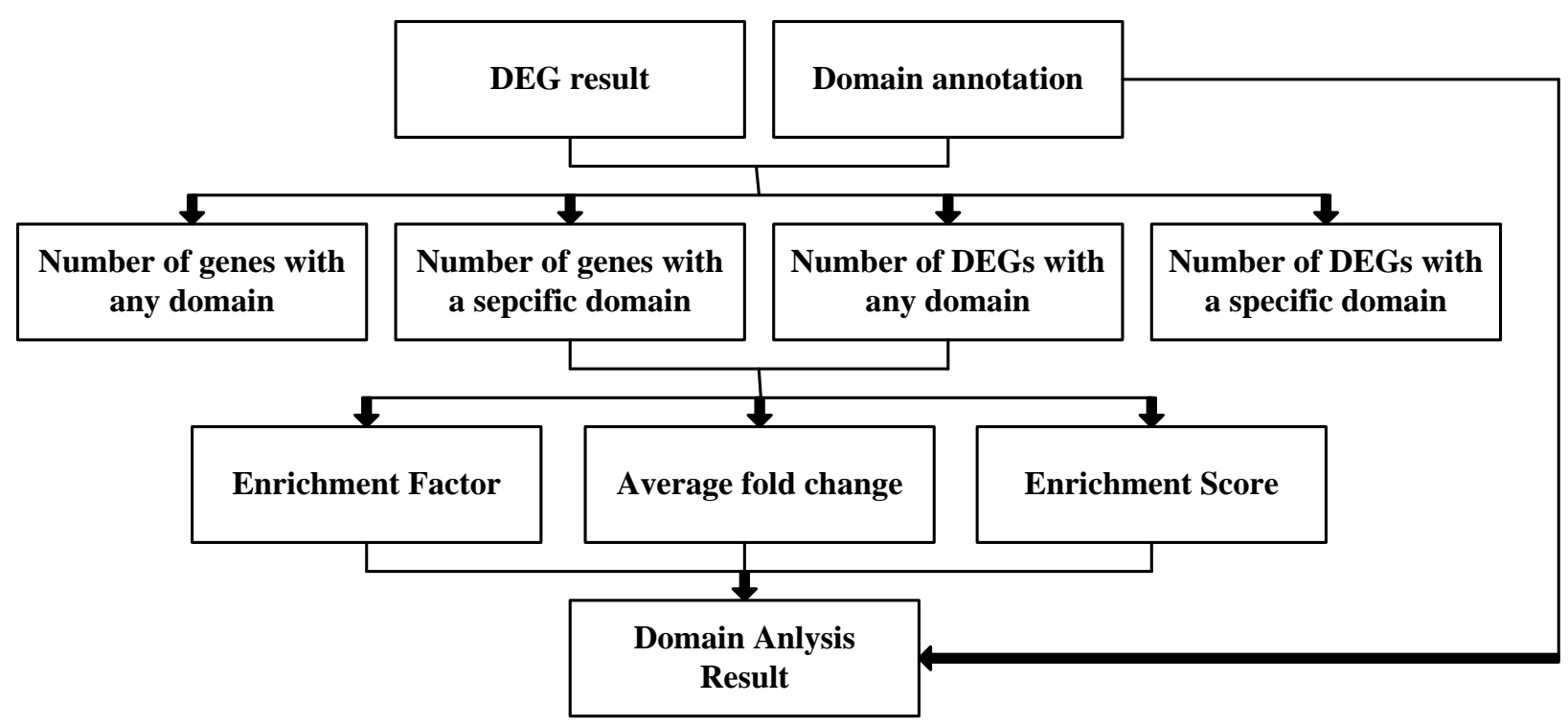

Figure 3: Domain Enrichment Flowchart

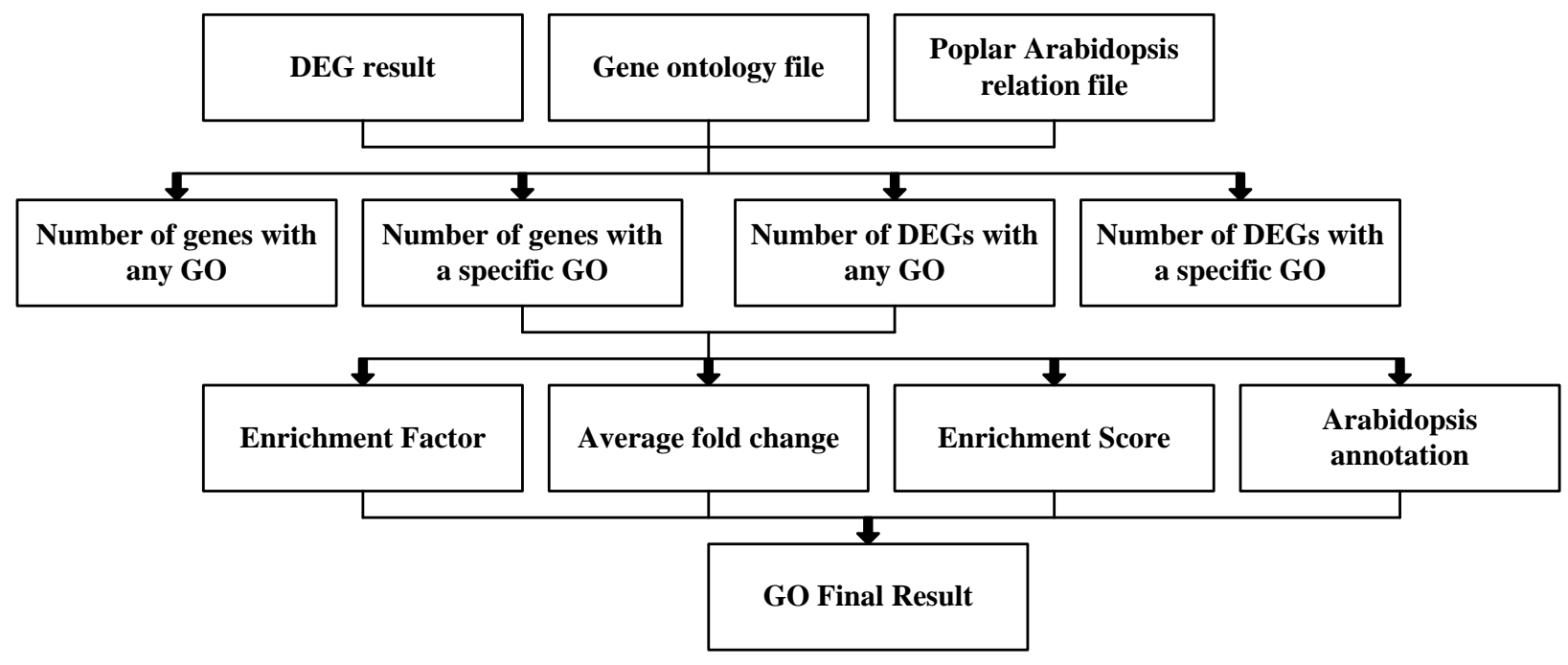

Figure 4: GO Enrichment Analysis Flowchart

\subsection{Roles and tasks}

I was responsible for the development of command-line pipeline programs and the web tools. I also performed the data analyses after getting results from pipeline program. Chapter 2 introduces the usage and functions of various pipeline programs I developed. Chapter 3 shows the GO hierarchy analysis. Chapter 4 provides the usage of our online tool. 


\section{Chapter 2}

\section{Poplar Gene Expression Data Analysis Pipeline}

\subsection{Identification of DEGs}

To identify DEGs, the pipeline perform the following procedures:

\subsection{1: Trimming part of genes}

Genes with high expression values and standard deviations usually produce significant biological effect. Thus we exclude those genes with lower expression values and smaller standard deviation at the very beginning.

The DEG pipeline ranks all the genes by their average expression values and standard deviations separately in descending order, and then combines these two ranks and calculates the average rank for each gene. The pipeline does the trimming according to this average rank.

Trimming gene is optional. Users can specify the cut-off value represents the trimming percentage. For instance, 0.2 represents trimming 20 percent genes.

\subsection{2: Calculating p-values for each gene}

We integrate Rank Product into our pipeline for identifying DEGs. Rank Product is a non-parametric statistic method for the detection of DEGs for microarray data $^{[3]}$. It is based on the rankings of fold changes. The formula for calculating the rank product is as follows:

$$
\operatorname{RankProduct}(\mathrm{g})=\left(\prod_{i=1}^{k} r_{g, i}\right)^{1 / k}
$$

Where $\mathrm{g}$ represents a specific gene, $\mathrm{n}$ represents the number of all the genes; $\mathrm{k}$ represents the number of replicates; $r_{g, i}$ represents the rank of ith replicate of gene $g$.

This method assumes that the orders of all the genes are random, i.e. the probability of finding a specific gene among the top $r$ of $n$ genes list equals to $r / n$. 
Rank Product method is fast and simple. It provides a straightforward and statistically stringent way to determine the significance level for each gene and reliable in high noisy data ${ }^{[3]}$.

Besides Rank Product, we also implemented two additional methods to identify the DEGs, they are Weight Average Difference (WAD) and moderated Tstatistics $(\operatorname{modT})$.

WAD is a fold-change-based method. It uses the average difference and relative average signal intensity so that DEGs are highly ranked on the average for the different conditions. ${ }^{[6]}$

For an individual gene $\mathrm{i}$ in time point $\mathrm{j}$, we need to calculate the average difference of this gene first. The average difference of gene $i$ in time point $j$ is calculated as followings:

$$
A D_{i j}=A V G_{i j}^{\text {control }}-A V G_{i j}^{\text {treatment }}
$$

$A V G_{i j}^{\text {control }}$ represents the average log signal of all the control replicates belong to gene $\mathrm{i}$ in time point $\mathrm{j}, A V G_{i j}^{\text {treatment }}$ represents the average log signal of all the treatment replicates belong to gene $\mathrm{i}$ in time point $\mathrm{j}$.

Average log signal intensity $w_{i j}$ is used to evaluating the average difference of all the replicates of gene $i$ in time point $j$.

$$
w_{i j}=\frac{A V G_{i j}^{\text {vector }}-\min (\text { vector })}{\max (\text { vector })-\min (\text { vector })}
$$

$A V G_{i j}^{\text {vector }}$ represents the average log signal of all the replicates belonging to gene $i$ in time point $j$. Vector is used to store the all the replicates of gene $i$ in time point $\mathrm{j}$, and the result is calculated as follows:

$$
W A D_{i j}=A D_{i j} * w_{i j}
$$

The larger the $W A D_{i j}$, the more significant a differentially expressed a gene is. WAD statistics can be an alternative of rank product in defining DEGs.

The modT is considered as reformulated posterior odds statistics, in which posterior residual standard deviations are used in place of ordinary standard deviations. ${ }^{[7]}$ Moderated t-statistic follows a t-distribution with augmented degrees of freedom, and it generates a more predictable ranking of genes compared with ordinary t-statistics. 
We also implemented the modT method to calculate the rankings of poplar genes in each time point. The larger the modT value, the more significant a differentially expressed a gene is. It is also an alternative of Rank Product in defining DEGs.

Other than these three methods, we also attempted to use ANOVA to identify DEGs. However, this method is too time-consuming and we finally decided not to integrate it.

\subsection{3: Trimming genes with larger $p$-values}

The selection of DEGs is based on the result of Rank Product method. We get pvalue for each remaining genes in step 1. We sort genes based on p-values in ascending order and trim those genes with p-value larger than 0.1 .

\subsection{4: P-value correction}

As dataset becomes larger, false positives get increased in DEG list. In order to make the false positive genes under control, we had to apply a multiple testing correction and calculate the corrected p-values.

Multiple methods are available for multiple testing of p-values. We chose Benjamini and Hochberg false discovery rate correction method ${ }^{[8]}$ in our program. This p-value correction method is the least stringent compared to other methods

such as Bonferroni and Westfall and young Permutation. ${ }^{[9] .}$

Benjamini Hochberg FDR multiple correction method calculates corrected pvalue as follows:

1. Sort the original p-values of all genes in ascending order.

2. Calculate corrected p-value by using following formula:

$$
\text { Corrected } \mathrm{p} \text {-value }=\text { Original } \mathrm{p} \text {-value } * \mathrm{n} / \mathrm{r}
$$

$\mathrm{r}$ stands for the rank of the gene and $\mathrm{n}$ stands for the total number of genes.

\subsection{5: Selection of DEGs}

After calculating corrected p-values, we can select DEGs. In our program, all the genes with corrected p-value smaller than 0.05 are defined as DEGs by default. This threshold is believed to be reasonable and also suggested by Benjamini Hochberg FDR correction method. Thus, the false discovery rate of the DEG list is limited to $5 \%$. However, users can also specify their own threshold. 
User must normalize their microarray expression data before using the pipeline to do analysis. Our pipeline tool takes the normalized microarray expression gene data file as input. The format of an example microarray expression gene data file is shown in Figure 5:

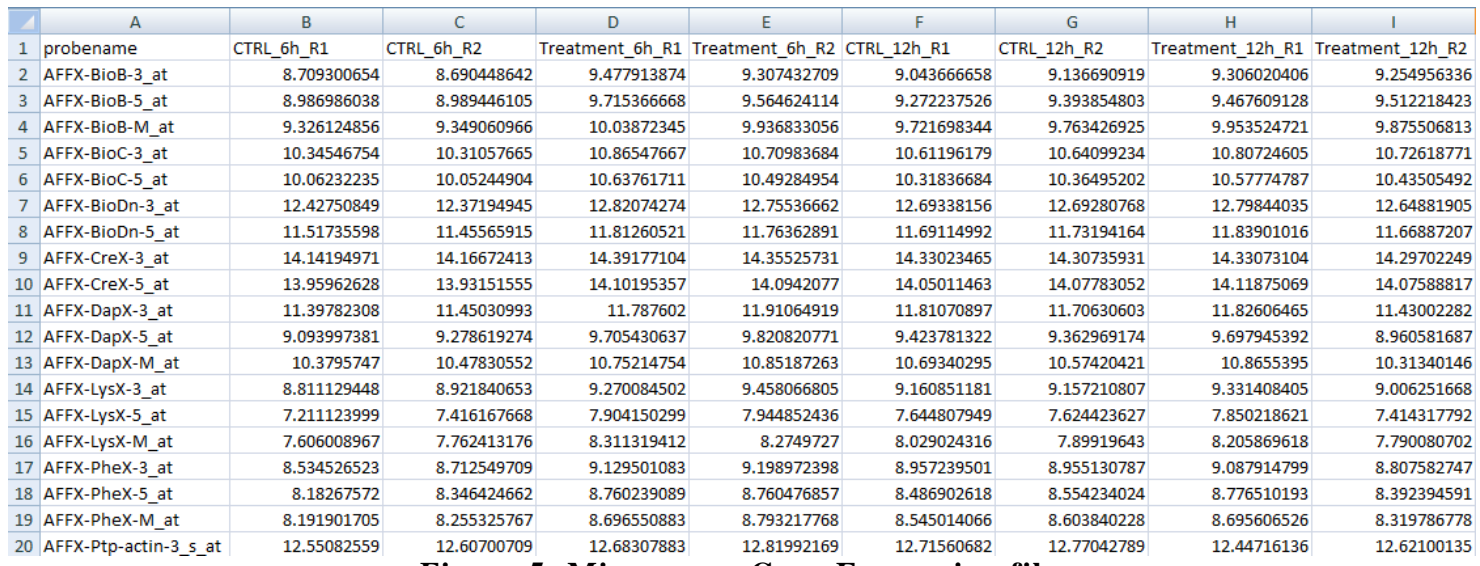

Figure 5: Microarray Gene Expression file

We have the following restrictions for the input microarray gene expression file:

a) The input file should be a tab-delimited file.

b) If we have multiple time points in the microarray gene expression file, the number of control and treatment replicates should be the same.

c) In each time point, we should have at least two replicates for control and also for treatment.

d) Row 1 provides the name of each column. The names of control replicates should start with "c" or " $\mathrm{C}$ " and the names of the treatment replicates should start with " $t$ " or "T".

Running command:

\$ perl ./script/DEG_pipeline.pl -p gene -i file1.txt -g file2.txt -t 0.2 -tp 6

- -p gene/pathway: 'gene' indicates we are doing DEG analysis and 'pathway' indicates we want to do pathway analysis.

- $\quad-i<$ file $>$ : Specifies the microarray gene expression file.

- - g $\langle$ file $>$ : Specifies the microarray gene annotation file.

- $-\mathrm{t}(0-1)$ : Specifies the trimming percentage of the original data introduced in 2.1 .1 ; the value should between 0 and 1 .

- -tp: Specifies the time point number, this number should be positive integer. 
The resulting file of a differentially expressed genes sample is shown in Figure 6:

\begin{tabular}{|c|c|c|c|c|c|c|c|c|c|c|}
\hline 4 & A & B & C & D & $E$ & $\mathrm{~F}$ & G & $\mathrm{H}$ & 1 & $\mathrm{~J}$ \\
\hline 1 & ProbelD & 1 Avg_ctrl & 1 Avg_treat & 1 FoldChange & 1 Rank_modT & 1 Rank_WAD & $1 \mathrm{RP}$ _origPvalue & $1 \mathrm{RP}_{\text {_correctedPvalue }}$ & 1 Rank_RP & $1 \mathrm{DEG}$ \\
\hline 3 & PtpAffx.224655.1.S1_s_at & 18.34824367 & 18.14094689 & -1.01142701 & 58154 & 55539 & 0.4466 & & & nonDEG \\
\hline 5 & Ptp.303.2.A1_at & 38.72076724 & 13.93961806 & 5 -2.77774951 & 1248 & 7735 & 0.0034 & 0.04283728 & 2078 & DEG \\
\hline 6 & PtpAffx.206393.1.S1_s_at & 3889.644684 & 673.9855423 & -5.771109971 & 2617 & 58 & $1.00 \mathrm{E}-04$ & 0.01149562 & 423 & DEG \\
\hline 7 & PtpAffx.218496.1.S1_at & 48.51263417 & 64.07073023 & 3. 1.320701943 & 22131 & 21249 & 0.1461 & & & nonDEG \\
\hline 8 & PtpAffx.224971.1.S1_at & 16.62052848 & 20.81897111 & 1.252605845 & 29675 & 30664 & 0.2022 & & & nONDEG \\
\hline 11 & PtpAffx.71036.1.S1_at & 76.5303036 & 62.25285574 & -1.229346071 & 24886 & 23578 & 0.17 & & & nonDEG \\
\hline 12 & PtpAffx.11727.1.A1_at & 132.4413342 & 640.0912355 & 4.833017117 & 2913 & 378 & 0 & 0 & 28 & DEG \\
\hline 13 & PtpAffx.10911.1.S1_at & 1585.534821 & 1236.5875 & -1.28218571 & 18556 & 11044 & 0.1354 & & & nonDEG \\
\hline 14 & PtpAffx.204364.1.S1_at & 433.2910001 & 329.6348552 & -1.314457477 & 29399 & 11286 & 0.1068 & & & nonDEG \\
\hline 15 & PtpAffx.20863.1.A1_at & 7742.467194 & 7004.539907 & -1.105349858 & 39874 & 20105 & 0.3026 & & & nonDEG \\
\hline 16 & Ptp.2730.1.S1_at & 71.25802974 & 123.5225711 & 1.733454763 & 8700 & 8391 & 0.0126 & 0.094314354 & 5324 & nonDEG \\
\hline
\end{tabular}

Figure 6: Sample DEG result file

Row 1 contains the column name. From column 2, the integer in the front of each column name indicates which time point that result belongs to. Column 2 provides the sum of unlogged expression values of all control replicates in that time point. Column 3 stands for the sum of unlogged expression values of all treatment replicates in that time point. Column 4 presents the fold change. Column 5 provides the rank for each sample from the moderated $\mathrm{T}$ method. Column 6 contains the ranks from the WAD method. Column 7 contains the pvalue results from the Rank Product method. Column 8 provides the corrected pvalue. Column 9 provides the ranks from the Rank Product method. Column 10 indicates whether the current gene is DEG or not in a selected time point.

\subsection{Identification of Differentially Expressed Pathways}

Metabolic pathways are series of chemical reactions occurring within a cell. In each pathway, a precursor chemical is modified by a series of chemical reactions to produce one or multiple chemicals. Usually enzymes encoded by multiple genes catalyze these reactions coordinately.

Gene set analysis evaluates the expression of a set of genes catalyzing biological pathways. ${ }^{[10]}$ In this analysis, users need to upload their matrix file that is used to provide the relations between pathways and genes. Figure 7 shows the format of a pathway matrix file. 


\begin{tabular}{|l|l|l|l|l|}
\hline & beta;-alanine biosynthesis I & beta;-alanine biosynthesis II & beta;-alanine biosynthesis III cis-zeatin biosynthesis \\
\hline probelD & 1 & 0 & 0 \\
\hline Ptp.6223.1.S1_s_at & 1 & 0 & 0 \\
\hline PtpAffx.24142.1.A1_at & 0 & 1 & 0 \\
\hline PtpAffx.208466.1.S1_s_at & 0 & 1 & 0 \\
\hline PtpAffx.208467.1.S1_at & 0 & 1 & 0 \\
\hline PtpAffx.218950.1.S1_at & 0 & 1 & 0 \\
\hline PtpAffx.223598.1.S1_at & 0 & 1 & 0 \\
\hline PtpAffx.3156.1.A1_s_at & 0 & 1 & 0 \\
\hline PtpAffx.51307.1.S1_s_at & 0 & 0 & 0 \\
\hline PtpAffx.144250.1.S1_s_at & 0 & 0 & 1 \\
\hline PtpAffx.144250.2.S1_at & 0 & 0 & 1 \\
\hline PtpAffx.219043.1.S1_at & 0 & 0 & 1 \\
\hline PtpAffx.95543.1.A1_at & 0 & 0 & 0 \\
\hline PtpAffx.129568.2.A1_a_at & 0 & 0 & 0 \\
\hline PtpAffx.126487.1.S1_s_at & 0 & 0 & 0 \\
\hline PtpAffx.201244.1.S1_at & 0 & 0 & 0 \\
\hline PtpAffx.205240.1.S1_at & 0 & 0 & 0 \\
\hline PtpAffx.206898.1.S1_at & 0 & 0 & 0 \\
\hline PtpAffx.249.331.S1_a_at & 0 & 0 & 0 \\
\hline PtpAffx.249.331.S1_at & & 0 & 0 \\
\hline
\end{tabular}

Figure 7: pathway matrix file

In the matrix file as shown, each row represents a poplar gene and each column represents a pathway. In this file 0 indicates the gene is not present in this pathway and 1 indicates the gene belongs to this pathway. In order to enable the pathway analysis using sample power, we combined the sample replicates for all the time points together prior to the analysis.

Significance Analysis of Microarray for Gene Sets (SAM-GS) was integrated into our pipeline for pathway enrichment analysis. ${ }^{[7]}$ Normally when we analyze whether a set of genes are associated with a phenotype, we measure to check whether the gene set consists of genes whose expression values continuously changed in one direction with the phenotype. Compared to GSEA (Gene Set Enrichment Analysis) method proposed by Mootha et al. ${ }^{[11]}$, SAM-GS is more sensitive, leading to the result of statistically significant gene sets more reliable.

We identified the differentially expressed pathways in the following steps:

\subsection{1: Calculate the fold change for each poplar gene}

Calculate the fold change for each individual poplar gene appears in the gene expression file in each time point separately. The calculation of fold change (FC) for given poplar gene $\mathrm{g}$ exists in time point $\mathrm{i}$ is as follows:

1. Calculate the sum of control replicates and treatment replicates in time point $i$. We refer them as sum_control, sum_treatment.

2. If sum_treatment $>=$ sum_control, $\mathrm{FC}=$ sum_treatment/sum_control.

If sum_treatment $<$ sum_control, FC $=-1 *$ sum_control/sum_treatment.

Negative FC indicates gene $\mathrm{G}$ in time point $\mathrm{i}$ is down-regulated, otherwise it is up-regulated. 


\subsection{2: Call SAM_GS program to evaluate all the pathways defined in pathway matrix file}

SAM_GS program calculates the p-value and corrected p-value for all the pathways base on the members in current pathway. The smaller the corrected $\mathrm{p}$ value, the more significant a pathway is.

\subsection{3: Calculate the average fold change for each pathway}

Suppose pathway $\mathrm{P}$ contains $\mathrm{n}$ genes, denoted as gene-1, gene-2, gene-3...gene-n.

a. For each pathway gene, if FC of such gene is larger than 1, subtract it by 1 ; if it is smaller than -1 , add 1 .

b. Add the resulting values of all the genes together. And then divided the sum by $\mathrm{n}$.

c. If the result for the previous step is a negative value, add -1. Otherwise add 1 . This resulting value is average fold change of the pathway.

Running command:

\$perl ./script/DEG_pipeline.pl -p pathway -i file1.csv -g anno/file2.csv

- -p gene/pathway: the option of 'pathway' indicates the analysis is to identify differentially expressed rather than gene

- $-\mathrm{i}\langle$ file>: Specifies the microarray gene expression file, this file should be CSV format, i.e. comma delimited file.

- $-\mathrm{g}\langle$ file $\rangle$ : Specifies the pathway matrix file, this file should be CSV format, i.e. comma delimited file.

The result file of pathway analysis is shown in Figure 8:

\begin{tabular}{|c|c|c|c|c|c|c|c|}
\hline 4 & A & B & C & D & E & $\mathrm{F}$ & G \\
\hline 1 & GS.stats.GS.name & GS.stats.GS.size & GS.stats.GS.p_value & GS.stats.GS.q_value & Sample genes & Avg_FC_1 & Avg_FC_2 \\
\hline 2 & beta...alanine.biosynthesis.I & 4 & 0.22 & 0.073552975 & 5 Ptp.6223.1.S1_s_at|PtpAffx.24142. & 1.123079328 & -1.10390131 \\
\hline 3 & beta..alanine.biosynthesis.II & 19 & 0 & & DtpAffx.208466.1.S1_s_at|PtpAffx. & -1.168863948 & -1.07359295 \\
\hline 4 & beta..alanine.biosynthesis.III & 4 & 0.04 & 0.019609118 & 8 PtpAffx.144250.1.S1_s_at|PtpAffx. & -2.132689441 & 1.184874118 \\
\hline 5 & cis.zeatin.biosynthesis & 2 & 0.04 & 0.019609118 & B PtpAffx.129568.2.A1_a_at|PtpAffx & K 1.112594437 & -1.136496832 \\
\hline 6 & de.novo.biosynthesis.of.uridine.5..monophosphate & 8 & 0.05 & 0.023712113 & 3 PtpAffx.126487.1.S1_s_at|PtpAffx. & -1.384438885 & -1.061765606 \\
\hline 7 & arginine.biosynthesis.II..acetyl.cycle. & 5 & 0.01 & 0.008639661 & 1 PtpAffx.220320.1.S1_at|PtpAffx.21 & $1-1.355483741$ & 1.06476692 \\
\hline 8 & ent.kaurene.biosynthesis & 3 & 0.1 & 0.040027787 & 7 PtpAffx.201731.1.S1_at|PtpAffx.20 & 0 1.034604294 & -1.052352464 \\
\hline 9 & S.adenosyl.L.methionine.cycle & 11 & 0.04 & 0.019609118 & 8 Ptp.49.1.S1_at|Ptp.52.1.S1_at|Ptp. & . -2.225956179 & 1.203897029 \\
\hline 10 & methionine.biosynthesis.II & 11 & 0.04 & 0.019609118 & B Ptp.49.1.S1_at|Ptp.52.1.S1_at|Ptp. & 1. -1.927389267 & 1.204607464 \\
\hline 11 & trans.lycopene.biosynthesis & 3 & 0.01 & 0.008639661 & 1 PtpAffx.222709.1.S1_at|PtpAffx.53 & $3-1.095462514$ & 1.099340811 \\
\hline 12 & trans.zeatin.biosynthesis & 1 & 0.03 & 0.01678088 & B PtpAffx.204470.1.\$1_at & -1.028142986 & 1.008461927 \\
\hline 13 & X1_4.dihydroxy.2.naphthoate.biosynthesis.II..plants. & 1 & 0.02 & 0.012927493 & 3 PtpAffx.216312.1.S1_at & -1.021671263 & 1.091815285 \\
\hline 14 & X2.ketoglutarate.dehydrogenase.complex & 4 & 0.06 & 0.026987807 & 7 Ptp.4041.2.S1_s_at|PtpAffx.151462 & $2-1.222636506$ & 1.067167905 \\
\hline 15 & abscisic.acid.biosynthesis & 18 & 0.13 & 0.049107683 & 3 PtpAffx.129974.1.S1_s_at|PtpAffx. & 1.14851028 & 3 - -1.199176464 \\
\hline 16 & IAA.biosynthesis.I & 3 & 0.09 & 0.037044584 & 4 PtpAffx.204443.1.S1_5_at|PtpAffx. & -1.365869846 & -1.032888906 \\
\hline 17 & abscisic.acid.glucose.ester.biosynthesis & 16 & 0.1 & 0.040027787 & 7 PtpAffx.146922.2.A1_at|PtpAffx.2C & (c-2.425752449 & 1.097892291 \\
\hline 18 & cytokinins.O.glucoside.biosynthesis & 30 & 0.04 & 0.019609118 & B Ptp.6751.1.A1_s_at|Ptp.6831.1.S1_ & -2.007517721 & -1.011844321 \\
\hline 19 & cytokinins.9.N.glucoside.biosynthesis & 30 & 0.04 & 0.019609118 & 8 Ptp.6751.1.A1_s_at|Ptp.6831.1.S1_ & -2.007517721 & -1.011844321 \\
\hline 20 & cytokinins.7.N.glucoside.biosynthesis & 30 & 0.04 & 0.019609118 & 8 Ptp.6751.1.A1_s_at|Ptp.6831.1.S1_ & -2.007517721 & -1.011844321 \\
\hline
\end{tabular}

Figure 8: pathway analysis result

In the output file, each row represents one pathway. Column A contains the pathway name. Column B gives the number or poplar genes within this pathway. 
Column C contains the p-value. Column D gives the q-value (corrected p-value). Column E shows the list of poplar genes current pathway corresponds to. The following column provides the average fold change of pathway in each time point.

\subsection{Domain Enrichment Analysis}

A protein domain is a conserved part or structure of protein sequence that can function independently from the rest of protein. Domains vary in length from between about 25 amino acids up to 500 amino acids in length. We downloaded the Interpro-scanner and associated databases to our server and ran it to analyze 73013 protein sequences and identified all protein domains contained in these sequences. Each domain in our analyses is represented by an Interpro ID. Domain annotation file in Figure 9 provides the corresponding relation between protein domains and poplar genes.

\begin{tabular}{|c|c|c|c|}
\hline 4 & A & B & c \\
\hline 1 & domain & description & ProbelD \\
\hline 2 & IPR000005 & Helix-turn-helix,sAraCstype & PtpAffx.223505.1.S1_at \\
\hline 3 & IPR000005 & Helix-turn-helix,sAraCstype & PtpAffx.218921.1.S1_at \\
\hline 4 & IPR000005 & Helix-turn-helix,sAraCstype & PtpAffx.215626.1.S1_at \\
\hline 5 & IPR000005 & Helix-turn-helix,sAraCstype & PtpAffx.220281.1.S1_at \\
\hline 6 & IPR000005 & Helix-turn-helix,sAraCstype & PtpAffx.215016.1.S1_at \\
\hline 7 & IPR000007 & Tubby,sC-terminal & PtpAffx.200078.1.S1_at \\
\hline 8 & IPR000007 & Tubby,sC-terminal & Ptp.1282.1.S1_at \\
\hline 9 & IPR000007 & Tubby,sC-terminal & Ptp.1963.1.S1_at \\
\hline 10 & IPR000007 & Tubby,sC-terminal & Ptp.2238.1.S1_at \\
\hline 11 & IPR000007 & Tubby,sC-terminal & Ptp.2238.1.S1_s_at \\
\hline 12 & IPR000007 & Tubby,sC-terminal & Ptp.5835.1.S1_at \\
\hline 13 & IPR000007 & Tubby,sC-terminal & PtpAffx.124217.1.S1_s_at \\
\hline 14 & IPR000007 & Tubby,sC-terminal & PtpAffx.146084.1.S1_at \\
\hline 15 & IPR000007 & Tubby,sC-terminal & PtpAffx.26405.3.A1_at \\
\hline
\end{tabular}

One protein domain is usually contained by multiple poplar genes. In this file Column A shows the Interpro ID of the protein, Column B gives the description of certain protein domain, Column C provides the poplar genes contain current domain.

The outcome of biological events in the cells and tissues is determined by the protein protein interaction through domains. The purpose of domain enrichment analysis is to identify the gene families that are thriving or over-represented in the data. Domain enrichment analysis is applied on the DEGs output from DEG pipeline. .

According to the protein domain annotation file we have, nearly half of the poplar genes have protein domains. We evaluate the enriched protein domains for each DEG list from various time points separately. The procedures in detail are as follows:

\subsection{1: Calculate genome size, genome number, sample size, and sample number}

In the program for protein domain enrichment analysis, we calculate enrichment score and enrichment factor to evaluate the degree of enrichment of a protein domain. To do this, we need to obtain four parameters. 
1. Genome size

Genome size represents the total number of poplar genes that have at least one protein domain. It can be obtained by scanning the domain annotation file.

\section{Genome number}

Genome number represents the number of poplar genes that have a specific protein domain.

\section{Sample size}

Sample size represents the number of DEGs in current time point that have at least one protein domain.

4. Sample number

Sample number represents the number of DEGs that have a specific protein domain.

The genome size is fixed once the domain annotation file is given. The sample size is fixed given the specific time point. The values of genome number and sample number vary in domains and time points.

\subsection{2: Calculate Enrichment Factor}

The enrichment factor is calculated by the following formula:

$$
E F_{\text {domain }}=\frac{\text { num_sample } / \text { num_genome }}{\text { sample_size } / \text { genome_size }}
$$

Enrichment factor is one statistics to measure the enrichment level.

\subsection{3: Calculate Enrichment Score}

Enrichment score is the other criterion to evaluate the degree of a protein domain is enriched. The enrichment score of a specific domain is calculated by the quintile function for hypergeometric distribution - phyper:

$$
\mathrm{ES}_{\text {domain }}=\operatorname{phyper}\left(\text { num }_{\text {sample }}, \text { num }_{\text {genome }}, \text { size }_{\text {genome }}-\text { num }_{\text {genome }}, \text { size }_{\text {sample }}\right)
$$

\subsection{4: Calculate Average Fold Change}

The average FC of a set of genes in a domain is also an important parameter for us to evaluate the activities. 
Calculating the average FC of a domain is similar with calculating the average fold change of pathway. Suppose domain $D$ is corresponding to $n$ DEGs $\left(D E G_{1}, D E G_{2}, \ldots, D E G_{n}\right)$, their fold changes are $F C_{1}, F C_{2}, \ldots, F C_{n}$. The fold change can only be up-regulated, i.e. the value is larger than 1 or down regulated, i.e. the value is smaller than -1 .

At first, we calculate the sum of fold changes of all the DEGs by implementing the following formula:

$$
S U M_{F C}=\sum_{i}^{N_{u p}}\left(F C_{u p_{i}}-1\right)+\sum_{j}^{N_{\text {down }}}\left(F C_{\text {down }_{j}}+1\right)
$$

Then we use the following formula to calculate the average fold change:

$$
A V G_{F C-D}=\left\{\begin{array}{l}
\frac{S U M_{F C}}{N_{u p}+N_{d o w n}}+1, S U M_{F C}>0 \\
\frac{S U M_{F C}}{N_{u p}+N_{\text {down }}}-1, S U M_{F C}<0
\end{array}\right.
$$

For example, if domain $\mathrm{D}$ is corresponding to $2 \mathrm{DEGs}$ and there fold changes in certain time point are 2.5, -3. $S U M_{F C}=(2.5-1)+(-3+1)=-0.5$, average fold change $A V G_{F C-D}=\left(-\frac{0.5}{2}\right)-1=-1.25$.

Running command:

\$perl script/DomainAnalysis_pipeline.pl -i DEG_result.txt -g domain_anno.txt -tp n

- $\quad-\mathrm{i}\langle$ file $\rangle$ : Specifies the DEG result file.

- - g <file>: Specifies the protein domain annotation file.

- $\quad$-tp n: Specifies the time point number.

The result of domain analysis pipeline is shown in Figure 10:

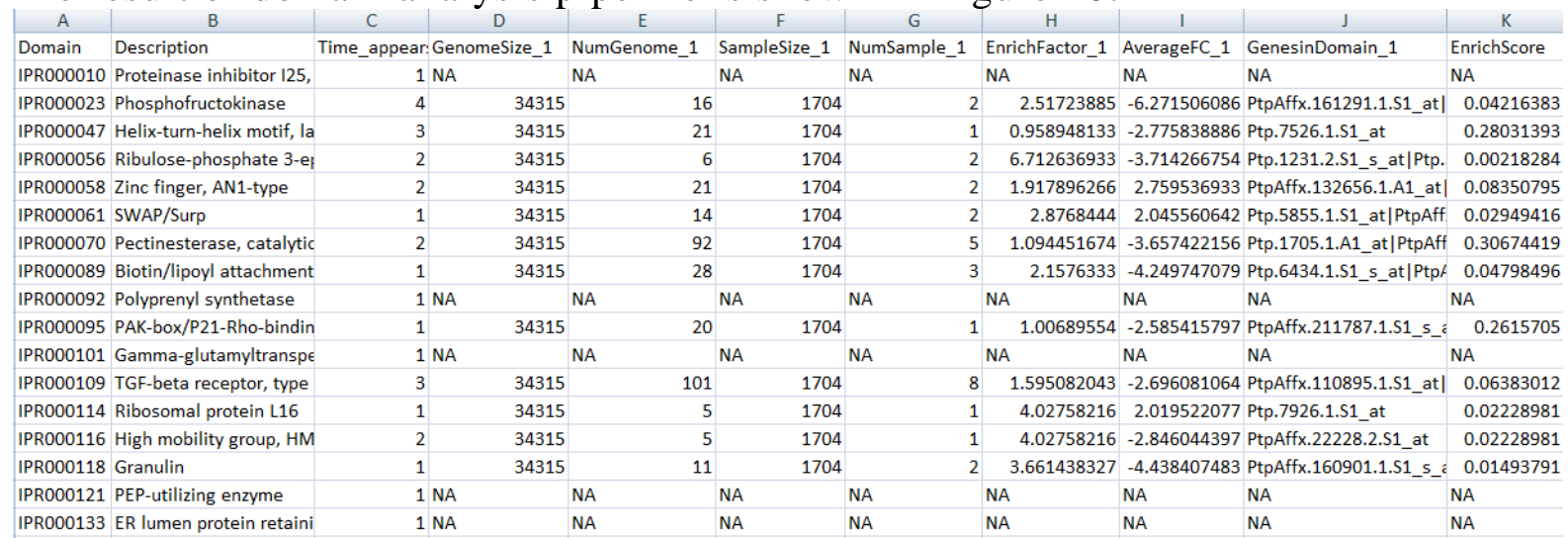

Figure 10: Result of protein domain analysis

In the output of protein domain analysis, Column A contains the Interpro ID. Column B gives the description of domain specified in column 1. Column $\mathrm{C}$ contains the number of times a certain domain's enrichment score is no larger than 0.05 among all time points. In the following columns one can find the 
values of genome size, genome number, sample size, sample number, enrichment factor, enrichment score and average fold change. A number of DEGs corresponds to the each domain is also shown in the result. If NA is list in all the parameters in a certain domain, then such domain is not possessed by any DEG in current time point.

We can identify the common domains and unique domains from the result of the domain analysis. Common domains refer to those protein domains with enrichment score smaller than 0.05 at all the time points. Unique domains refer to those protein domains with enrichment score smaller than 0.05 at only one time point.

\subsection{GO enrichment Analysis}

GO is a major bioinformatics initiative to standardize the representation of gene and gene product attributes across all species. ${ }^{[12]}$

In more details, the GO project aims at:

1. Maintain and develop its controlled vocabulary of gene and gene product attributes;

2. Annotate genes and gene products, and assimilate and disseminate annotation data;

3. Develop tools in order to facilitate the creation, maintenance as well as the usage of ontologies;

Since there is no universal standard terminology in biology and related domains, and term usages may be specific to a species, research area or even a particular research group. This makes communication and data sharing more difficult. The GO provides ontology of defined terms representing gene product properties. The ontology covers three domains:

1. Cellular component, the parts of a cell or its extracellular environment, where a gene product functions.

2. Molecular function, the elemental activities of a gene product at the molecular level, such as binding or catalysis;

3. Biological process, operations or sets of molecular events with a defined beginning and end involved by a gene product, pertinent to the functioning of integrated living units: cell, tissues, organs, and organisms.

Each of these domains is represented by a directed acyclic graph with one source. Cellular component, molecular function, and biological process are the sources of these three graphs. Each node in the graph is a term that represents a sub 
domain. When we have a gene list, we can examine the GO terms associated with the short gene lists and get some ideas about which molecular function, cellular component or biology process is enriched in the associated gene list.

In the GO enrichment analysis, we tried to identify the GO terms related to DEGs in each time point. However, the current databases do not contain the relations between GO terms and poplar genes. However in the GO database, there is an Arabidopsis thaliana annotation file - TAIR. This file provides the relations between GO terms and Arabidopsis genes, shown in Figure 11:

\begin{tabular}{|c|c|c|c|c|c|c|c|}
\hline TAIR & locus:2193997 & P40 & GO:0000028 & $\mathrm{P}$ & AT1G72370 & AT1G72370|P40|AP40| protein & TAIR:locus:2193997 \\
\hline TAIR & locus:2058324 & AT2G04390 & GO:0000028 & $\mathrm{P}$ & AT2G04390 & AT2G04390|T1O3.20|T1 protein & TAIR:locus:2058324 \\
\hline TAIR & locus:2051229 & AT2G05220 & GO:0000028 & $\mathrm{P}$ & AT2G05220 & AT2G05220|F5G3.12|F5 protein & TAIR:locus:2051229 \\
\hline TAIR & locus:2084988 & RPSAb & GO:0000028 & $P$ & АT3G04770 & AT3G04770|RPSAb|40s protein & TAIR:locus:2084988 \\
\hline TAIR & locus:2075735 & АТЗG10610 & GO:0000028 & P & АТЗG10610 & AT3G10610|F13M14.10 protein & TAIR:Iocus:2075735 \\
\hline TAIR & locus:2175428 & AT5G04800 & GO:0000028 & P & AT5G04800 & AT5G04800|MUK11.13 protein & TAIR:locus:2175428 \\
\hline TAIR & locus:2185485 & AT5G14850 & GO:0000030 & $\mathrm{F}$ & AT5G14850 & AT5G14850|T9L3.150|T: protein & TAIR:locus:2185485 \\
\hline TAIR & locus:504954755 & PNT1 & GO:0000030 & $\mathrm{F}$ & AT5G22130 & AT5G22130|PNT1|PEAI protein & TAIR:Iocus:504954755 \\
\hline TAIR & locus:2201786 & FATB & GO:0000036 & $\mathrm{F}$ & AT1G08510 & AT1G08510|FATB | fatty protein & TAIR:locus:2201786 \\
\hline TAIR & locus: 2206300 & mtACP2 & GO:0000036 & $\mathrm{F}$ & AT1G65290 & AT1G65290 $\mid$ mtACP2 $\mid \mathrm{m}$ protein & TAIR:locus: 2206300 \\
\hline TAIR & locus:2042331 & MTACP-1 & GO:0000036 & $\mathrm{F}$ & mitochondrial & | AT2G44620|MTACP1 $\mid \mathrm{N}$ protein & TAIR:gene:2042330 \\
\hline TAIR & locus:2042331 & MTACP-1 & GO:0000036 & $\mathrm{F}$ & AT2G44620 & AT2G44620|MTACP-1|^ protein & TAIR:locus:2042331 \\
\hline TAIR & locus:2090285 & FaTA & GO:0000036 & $\mathrm{F}$ & АТЗG25110 & AT3G25110|AtFaTA|Fa- protein & TAIR:locus:2090285 \\
\hline TAIR & locus:2123256 & AT4G13050 & GO:0000036 & $\mathrm{F}$ & AT4G13050 & AT4G13050|F25G13.140 protein & TAIR:locus:2123256 \\
\hline TAIR & locus:2181216 & ACP5 & GO:0000036 & $\mathrm{F}$ & AT5G27200 & AT5G27200|ACP5|acyl / protein & TAIR:locus:2181216 \\
\hline TAIR & locus:2142908 & AT5G35930 & GO:0000036 & $\mathrm{F}$ & & AT5G35930|AT5G35930 protein & TAIR:gene:2142907 \\
\hline TAIR & locus:2168968 & mtACP3 & GO:0000036 & $\mathrm{F}$ & mitochondrial & | AT5G47630|AT5G47630 protein & TAIR:gene:4010713302 \\
\hline TAIR & locus:2168968 & mtACP3 & GO:0000036 & $\mathrm{F}$ & mitochondrial & | AT5G47630|AT5G47630 protein & TAIR:gene:2168967 \\
\hline
\end{tabular}

Figure 11: Gene Association file - TAIR

After obtaining the relations between GO terms and Arabidopsis genes, we map Arabidopsis genes to poplar genes according to our poplar gene annotation file. Figure 12 shows the mapping relations between poplar genes and Arabidopsis genes.

\begin{tabular}{|l|c|}
\hline \multicolumn{1}{|c|}{ A } & B \\
\hline AFFX-BioB-M_at & AT2G43360 \\
\hline AFFX-Ptp-gapdh-3_at & AT1G12900 \\
\hline AFFX-Ptp-r2-Ec-bioB-3_s_at & AT2G43360 \\
\hline AFFX-r2-EC-bioB-M_at & AT2G43360 \\
\hline Ptp.1003.1.A1_at & AT1G67060 \\
\hline Ptp.1006.1.A1_at & AT1G74750 \\
\hline Ptp.101.1.S1_at & AT1G61667 \\
\hline Ptp.1011.1.S1_s_at & AT3G11780 \\
\hline Ptp.1042.1.A1_at & AT1G79660 \\
\hline
\end{tabular}

Figure 12: Relations between poplar genes and Arabidopsis genes

Arabidopsis genes and poplar genes are homologous, i.e. they are functionally similar. Thus we can generate a mapping table to reveal their relationships. GO enrichment analysis evaluate GO terms of DEGs at each time point. The procedures in details are as follows:

\subsection{1: Generate a list of Arabidopsis genes of DEGs in each time point}


By consulting the Arabidopsis - poplar homolog gene chart released by Phytozome.com, in 2012, we generate a file containing a list of Arabidopsis genes corresponds to DEGs in each time point. Two or more poplar genes may have the same Arabidopsis homolog gene.

\subsection{2: Call TermFinder to generate GO terms given the list of Arabidopsis genes}

We use TermFinder ${ }^{[13]}$ to identify the enriched GO terms from a list of DEGs. The module can be downloaded from CPAN.

TermFinder outputs a set of GO terms based on the list of Arabidopsis genes. The result is based on the background database, gene numbers in the list, the frequency of GO terms are annotated across the provided gene list. Like the domain analysis, the P-value is also calculated by the function of the hypergeometirc distribution. ${ }^{[13]}$ The method also calculates the corrected p-value for each GO by applying Bonferroni correction method. The result of TermFinder is shown in Figure 13:

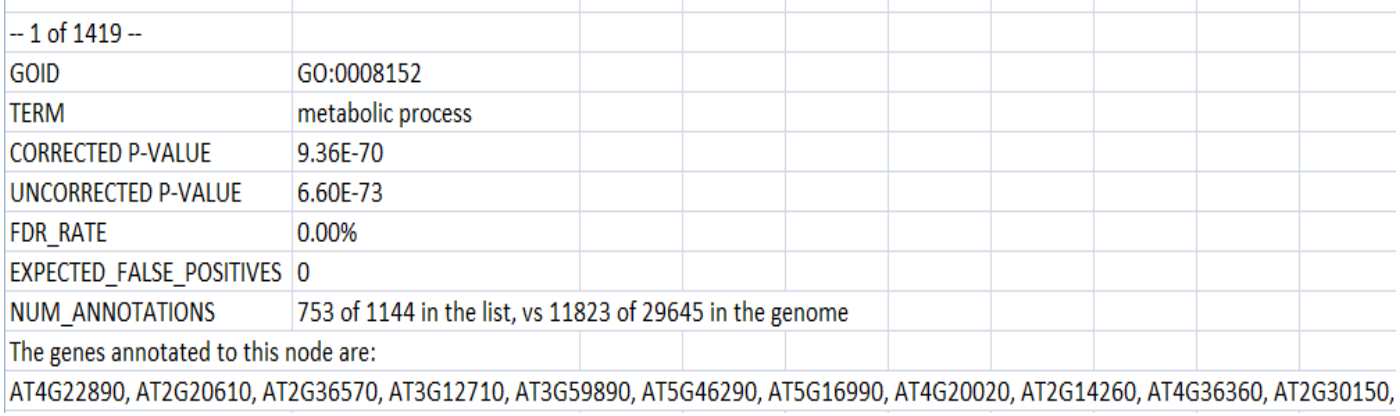

Figure 13: Result from TermFinder

\subsection{3: Mapping the Arabidopsis gene list of each GO back to poplar genes, and calculating the genome size, genomic gene number, sample size, sample number}

From the sample shown in Fig 2-9 we can see a list of Arabidopsis genes associated with a GO term. Now we use this list map back to poplar genes, and then calculate the average fold changes for each GO. We also need to get the genome size, genome number, sample size and sample number as domain analysis to calculate the p-value for each GO.

1. Genome size 
Genome size represents the total number of poplar genes exist in DEG result file that corresponds to at least one GO term listed in the result of TermFinder in current time points.

2. Genomic gene number

Genomic gene number represents the number of poplar genes that associated with current GO term.

\section{Sample size}

Sample size represents the number of DEGs corresponds to at least one GO term specified in the result of TermFinder in current time point.

\section{Sample number}

Sample number represents the number of DEGs associated with the current GO term.

Once we get these four numbers, we can calculate the enrichment factor and enrichment score as what we did for protein domain analysis. We can also calculate the average fold change by mapped poplar genes associated with a specific GO term. Here we calculate two average fold changes of all genes associated with each GO in each time point. Average fold change 1 is calculated by mapping the Arabidopsis genes back to DEGs in current time point.. Average fold change 2 is calculated by mapping the Arabidopsis genes back to whole poplar gene set. The calculation is also the same as which specified in domain enrichment analysis.

\subsection{4: Appending the annotation of Arabidopsis genes to the result}

In GO analysis, we also append the annotation of Arabidopsis genes to the result. The Arabidopsis annotation file is shown in Figure 14:

\begin{tabular}{|c|c|c|}
\hline A & B & \\
\hline Name & Type & Short_description \\
\hline AT1G01010 & protein_coding & ANAC001 (Arabidopsis NAC domain containing protein 1); transcription factor \\
\hline AT1G01020 & protein_coding & ARV1 \\
\hline AT1G01040 & protein_coding & DCL1 (DICER-LIKE 1); ATP-dependent helicase/ double-stranded RNA binding / protein binding / ribonuclease III \\
\hline AT1G01046 & mirna & MIR838a; miRNA \\
\hline AT1G01050 & protein_coding & AtPPa1 (Arabidopsis thaliana pyrophosphorylase 1); inorganic diphosphatase \\
\hline AT1G01070 & protein_coding & nodulin MtN21 family protein \\
\hline AT1G01073 & protein_coding & unknown protein \\
\hline AT1G01080 & protein_coding & $33 \mathrm{kDa}$ ribonucleoprotein, chloroplast, putative / RNA-binding protein $\mathrm{cp} 33$, putative \\
\hline AT1G01090 & protein_coding & PDH-E1 ALPHA (PYRUVATE DEHYDROGENASE E1 ALPHA); pyruvate dehydrogenase (acetyl-transferring) \\
\hline AT1G01100 & protein_coding & $60 \mathrm{~S}$ acidic ribosomal protein $\mathrm{P} 1$ (RPP1A) \\
\hline AT1G01110 & protein_coding & IQD18 (IQ-domain 18) \\
\hline
\end{tabular}

Figure 14: Annotation file of Arabidopsis genes 


\subsection{5: Identification of common GO terms and Unique GO terms}

A common GO term is the one enriched in all time points. A unique GO term is defined as the one enriched in only one time point. After getting the results from each time point, we do a summation and generate two additional files for common GO terms and unique GO terms.

In order to show the result of common GO terms and unique GO terms more, we also use the barplot function in $\mathrm{R}$ to render the average fold change. Figure 15 shows average fold changes of unique GO terms in one time point generated by vertical barplot.
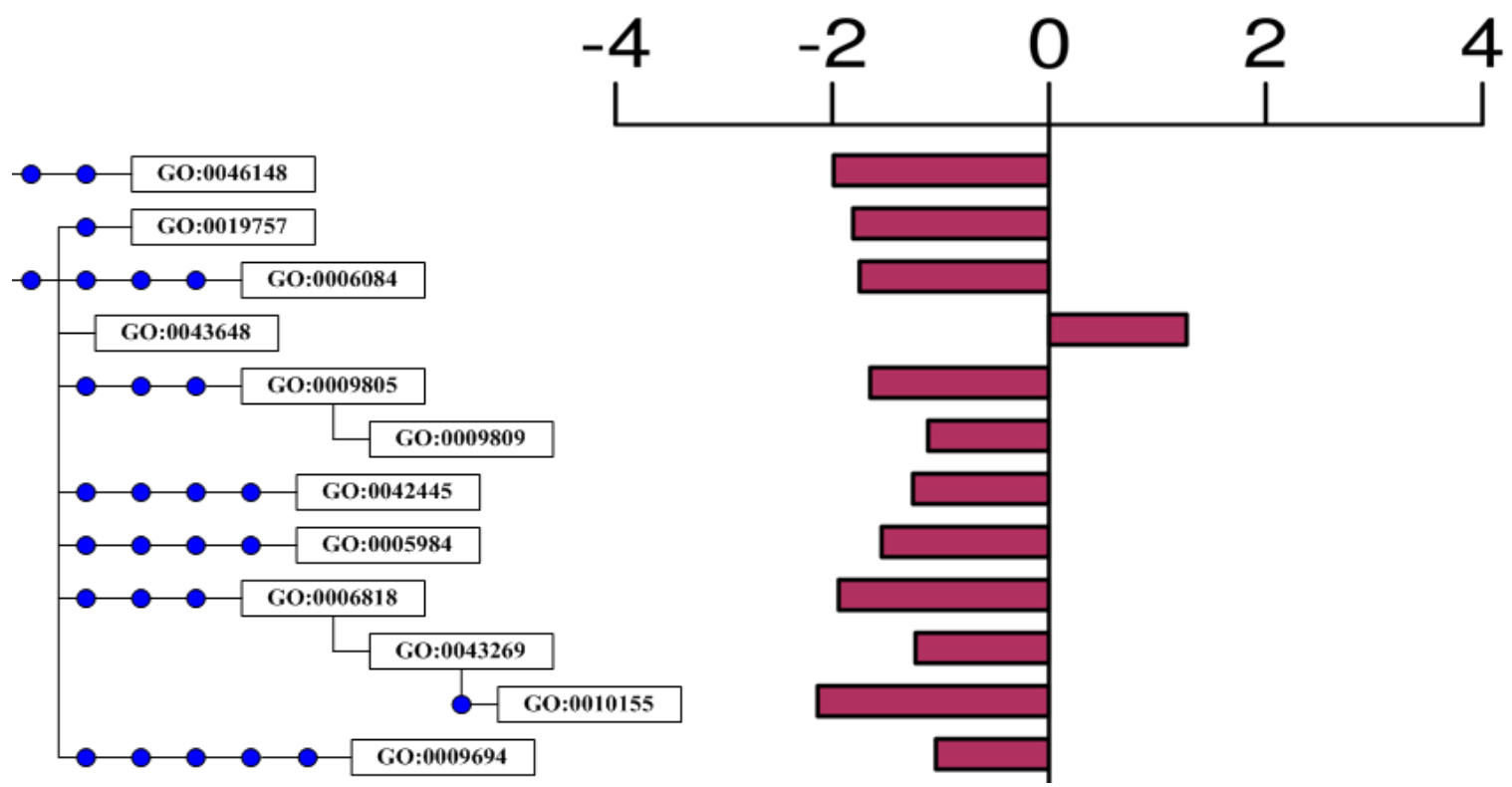

Figure 15: Unique GO terms rendered by vertical barplot

Running command:

\$ perl ./script/GO-Term_pipeline.pl -i DEG_result.txt -g relation.txt -tp n -e TAIR.txt n gene_ontology.obo

- $-\mathrm{i}\langle$ file $\rangle$ : Specifies the DEG output file

- $\quad$-g $\langle$ file $>$ : Specifies file provides relations between poplar genes, Arabidopsis genes and GO terms

- $-\mathrm{e}\langle$ file $\rangle$ : Specifies the annotation file of Arabidopsis genes

- $-n\langle$ file $\rangle$ : Specifies the GO ontology file

- $\quad$-tp n: Specifies the time point number

The output of GO enrichment analysis is shown in Figure 16: 


\begin{tabular}{|c|c|c|c|c|c|c|c|c|c|}
\hline Timepoint & GOID & GenomeSize & NumGenome & SampleSize & Numsample & Sample Poplar genes & Enrichment Factor & Enrichment Score & Term_Type \\
\hline 1 & I GO:0008152 & 22087 & 1341 & 1276 & 139 & Ptp.5042.3.S1_s_at|Ptp & 1.794205069 & $3.96 \mathrm{E}-12$ & $P$ \\
\hline 1 & 1 GO:0009987 & 22087 & 22 & 1276 & 1 & PtpAffx.144144.1.S1_s_ & 0.786798233 & 0.365729 & $P$ \\
\hline 1 & I GO:0005975 & 22087 & 552 & 1276 & 80 & PtpAffx.6111.2.S1_a_at & 2.508632048 & $8.24 \mathrm{E}-15$ & $P$ \\
\hline 1 & I GO:0044237 & 22087 & 90 & 1276 & 13 & PtpAffx.135595.1.S1_at & 2.500269941 & 0.00062891 & $P$ \\
\hline 1 & GO:0006091 & 22087 & 146 & 1276 & 2 & PtpAffx.220191.1.S1_at & 0.237117276 & 0.9917614 & $P$ \\
\hline 1 & GO:0006006 & 22087 & 25 & 1276 & 9 & PtpAffx.308.2.A1_x_at| & 6.231442006 & $5.88 \mathrm{E}-07$ & $7 \mathrm{P}$ \\
\hline 1 & GO:0055114 & 22087 & 1527 & 1276 & 176 & Ptp.6442.1.S1_at|Ptp.6: & 1.995077118 & $1.06 \mathrm{E}-19$ & $P$ \\
\hline 1 & $1 \mathrm{GO}: 0006007$ & 22087 & 138 & 1276 & 22 & PtpAffx.135595.1.S1_at & 2.759495252 & 4.14E-06 & $\mathrm{P}$ \\
\hline 1 & I GO:0042221 & 22087 & 4 & 1276 & 3 & Ptp.4373.1.S1_x_at|Ptp & 12.98217085 & 1.11E-05 & $\mathrm{P}$ \\
\hline 1 & GO:0019752 & 22087 & 19 & 1276 & 2 & Ptp.3400.1.S1_s_at|Ptp & 1.822059066 & 0.09348406 & $\mathrm{P}$ \\
\hline 1 & I GO:0006520 & 22087 & 82 & 1276 & 12 & PtpAffx.156807.1.S1_at & 2.533106507 & 0.000808222 & $\mathrm{P}$ \\
\hline 1 & GO:0046686 & 22087 & 733 & 1276 & 122 & Ptp.4195.1.S1_at|PtpAf & 2.880991075 & $2.86 \mathrm{E}-27$ & $7 \mathrm{P}$ \\
\hline 1 & 1 GO:0000096 & 22087 & 77 & 1276 & 8 & PtpAffx.83673.1.A1_at | & 1.798395961 & 0.03304748 & $P$ \\
\hline 1 & $1 \mathrm{GO}: 0008652$ & 22087 & 118 & 1276 & 13 & PtpAffx.83673.1.A1_at & 1.906985548 & 0.00815794 & $P$ \\
\hline
\end{tabular}

Figure 16: GO enrichment analysis result

In the output of GO enrichment analysis, Column 1 shows the time point to which this GO term belongs to. Column 2 gives the GOID. Column 3 provides the genome size. Column 4 provides the genomic gene number. Column 5 provides the sample size. Column 6 provides the sample number. Column 7 provides the list of DEGs correspond to the certain GO in current time point, followed by enrichment factor and enrichment score. Column 10 shows the type of current GO, "P" stands for biological process, "C" stands for cellular component, and "F" stands for molecular function. We still have several columns that are not shown up, which provide the $\mathrm{p}$-value, corrected $\mathrm{p}$-value and two averaged fold changes.

The Common GO result file is shown in Figure 17:

\begin{tabular}{|c|c|c|c|c|c|c|c|c|c|c|c|c|c|c|c|}
\hline A & B & c & D & $\mathrm{E}$ & $\mathrm{F}$ & G & $\mathrm{H}$ & I & J & $\mathrm{k}$ & L & M & $\mathrm{N}$ & 0 & 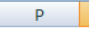 \\
\hline GO & Type & Term & Definition & 1 & 1 Time & 2 Timepoin & a Time & ATimepoir & Timepo & Timepoint & Timepoi & Timepoi & Timepoir & Timepoi & 10 \\
\hline GO:0009607 & Biological Process & response to & Any process & $\leq 2.21898523$ & -2.5825452 & 2.55400403 & -2.0249169 & -1.9707369 & 1.178478 & -2.50429 & 5.760706 & 1.828988 & 4.342654 & 1.808944 & -1.34673 \\
\hline GO:0006810 & Biological Process & transport & The directe & -2.4807625 & -3.1761805 & -1.0436531 & -2.9307502 & -1.7663082 & -5.62438 & -2.80612 & 1.908426 & 1.594501 & 1.18023 & 186858 & 1.55063 \\
\hline GO:0006950 & Biological Process & response to & Any & $\leq-1.1064286$ & 2.65277155 & 2.01745641 & -1.4625205 & 2.61855304 & -2.07774 & -3.12935 & 4.170214 & 1.775278 & 2.623059 & 310914 & 035952 \\
\hline GO:0055114 & Biological Process & oxidation-r & A metabolic & c -3.2822484 & 2.29997772 & 1.59875757 & 2.90702868 & 1.20993129 & 1.551408 & -3.18921 & 2.420716 & 1.708979 & 2.611416 & 1.162618 & 1.651754 \\
\hline GO:0051707 & Biological Process & espo & An & $=-2.9$ & 3.43227625 & 1.07758614 & 6.05 & -1.1451184 & -1.95449 & -2.50233 & 5.760706 & 1.822592 & 4.370659 & 1.808944 & 1.34673 \\
\hline GO:0009698 & Biological Process & phenylprop & The chemici & -3.6223225 & 1.31330276 & -2.1849499 & 3.06616661 & -1.8697484 & 3.820421 & -3.22545 & 7.580631 & -1.24299 & 5.336261 & -1.37046 & 2.36673 \\
\hline GO:0048046 & Cellular Components & apoplast & The cell me & -4.3315426 & 4.84655959 & 1.4580909 & 1.65987549 & -1.3546381 & -1.72272 & -4.33154 & 4.84656 & 1.458091 & 1.659875 & -1.35464 & -1.72272 \\
\hline GO:0005576 & Cellular Components & extracellula & The space $\mathrm{e}$ & -5.4037481 & 1.43531534 & 1.83447673 & 2.34141026 & -1.9120985 & -3.01804 & -4.49456 & 3.606107 & 1.462853 & 1.841239 & -1.43769 & -1.91702 \\
\hline GO:0005618 & Cellular Components & cell wall & The rigid or & -4.2932383 & 4.44663995 & 1.42204436 & 2.48132468 & -2.0762415 & -1.63939 & -4.13838 & 4.476576 & 1.26394 & 2.290695 & -2.06295 & -1.68014 \\
\hline GO:0003824 & Molecular Function & catalytic act & Catalysis of & -3.5012338 & 5.70548398 & 1.64631331 & 2.31646287 & 2.13699584 & -1.5176 & -3.23428 & 2.978361 & 1.122578 & 1.57915 & -1.29557 & -1.22188 \\
\hline GO:0016491 & Molecular Function & oxidoreduc & Catalysis of & -3.6439985 & 3.3042237 & 1.33008578 & 3.55277769 & 2.01260152 & 2.016899 & -3.2423 & 958537 & 1.648365 & 286023 & .156487 & .683157 \\
\hline 169 & Molecular Fur & tio & ilnt & $:-5.6$ & 2.7 & 768 & 73 & 27 & -2.508 & 21 & 059 & 232 & 1853 & 393 & 247293 \\
\hline GO:0046872 & Molecular Function & metal ion b & Interacting: & $:-2.2817767$ & 2.41174993 & -1.2701693 & -1.2647791 & 3.73785295 & -2.45113 & -3.15879 & 4.387965 & 1.214114 & 2.557244 & 1.09442 & 1.32043 \\
\hline
\end{tabular}

Figure 17: Result file of Common GO terms

The result file of common GO terms provides the type, the description as well as the two average fold changes among all the time points of a certain common GO. Users can check whether it is up-regulated or down-regulated in each time point conveniently.

The Unique GO result file is shown in Figure 18: 


\begin{tabular}{|c|c|c|c|c|c|}
\hline type & Timepoint & Term & Definition & Average_FC1 & Average_FC2 \\
\hline GO:0042023 Biological Process & & 3 DNA endoreduplic & c Regulated re-replication & -2.62973378 & $\begin{array}{ll}3 & -2.62973378\end{array}$ \\
\hline GO:0019760 Biological Process & & 1 glucosinolate met: & t: The chemical reactions $\hat{c}$ & c $\quad-3.1213787$ & -3.65014878 \\
\hline GO:0010564 Biological Process & & 3 regulation of cell c & c Any process that moduli & li -2.90131872 & -2.67449966 \\
\hline GO:0008610 Biological Process & & 1 lipid biosynthetic । & IThe chemical reactions $\hat{c}$ & a -3.76623028 & $\begin{array}{ll}3 & -3.56326147\end{array}$ \\
\hline GO:0006006 Biological Process & & 1 glucose metabolic & $c$ The chemical reactions $\hat{c}$ & ć -2.48303573 & -3.55240421 \\
\hline GO:0009913 Biological Process & & 4 epidermal cell diffi & fi The process in which a $r$ & -2.25315269 & -1.76916467 \\
\hline GO:0006094 Biological Process & & 1 gluconeogenesis & The formation of glucost & fe -3.72571474 & -3.72571474 \\
\hline GO:0006091 Biological Process & & 1 generation of prec & c The chemical reactions $\hat{c}$ & c 2.149351458 & $3-3.74688293$ \\
\hline GO:0006817 Biological Process & & 4 phosphate ion trar & ir The directed movement & -2.25210164 & +-2.25210164 \\
\hline GO:0006098 Biological Process & & 1 pentose-phosphat & t The process in which glu & u -3.07718786 & -3.07099927 \\
\hline GO:0080167 Biological Process & & 1 response to karriki & ki Any process that results & -2.61763418 & $\begin{array}{ll}3 & -2.61763418\end{array}$ \\
\hline
\end{tabular}

Figure 18: Result file of Unique GO terms

In the result file, column 3 specifies which time point the current GO belongs to, followed by the short description and definition of current GO. This file also gives two average fold changes of current GO. 


\section{Chapter 3}

\section{GO Hierarchy Analysis}

\subsection{Different types of GO relations}

The ontologies of GO terms are structured as a directed acyclic graph with only one source, with GO terms represented by nodes and relations denoted by arcs as nodes in the graph. Like all the terms are categorized into three different classes, the relationships between GO terms can be classified into the following categories; part of relation; has part relation; regulates relation, negatively regulatory relation and positively regulatory relation.

1. is a relation: If we say $\mathrm{A}$ is $a \mathrm{~B}$, we indicate that $\mathrm{A}$ is a subtype of $\mathrm{B}$. For example, fatty acid binding is a lipid binding, mucosal tolerance induction is a mucosal immune response, or lyase activity is a catalytic activity.

2. part of relation: The part of relation is used to represent part-whole relationships in the GO. A part of relation would only be added between A and B if B is necessarily part of A, i.e. the presence of the B implies the presence of A. For example, autophagic vacuole assembly is part of macroautophagy.

3. regulates relation: If one process directly affects the manifestation or quality of another process, then we say the former process regulates the latter process. The target of the regulation can be a pathway, an enzymatic reaction, or it may be a quality, such as cell size or $\mathrm{PH}$.

4. positively regulates relation \& negatively regulates relation: These two relations are two sub-relations of regulates relation.

For a given GO, its relations to other GO terms can be found in the obo file. For example:

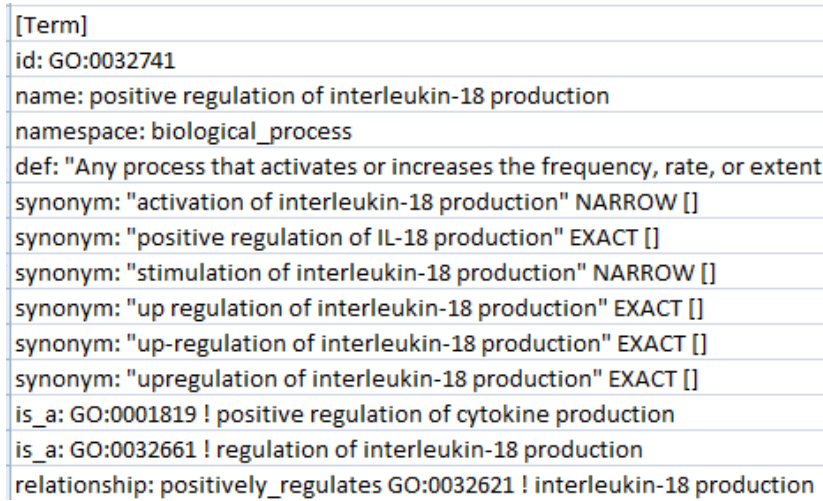

Figure 19: GO term example 
From Figure 19, we can see that GO:0032741 "is a" GO:0001819. GO:0032741 is a GO:0032661. GO:0032741 "positively regulates" GO:0032621.

There still exist some other GO relationships besides the fundamental ones we mentioned above, e.g. located in relation; adjacent to relation; has participant relation and so on. Our GO hierarchy analysis only takes the following 5 basic GO relations into consideration:

1 . is a relation

2. part of relation

3. regulates relation

4. negatively regulates relation

5. positively regulates relation

In our GO ontology analysis, we try to build a tree-structure graph to reveal the relations for a given list of GO terms. We make the source of the acyclic graph to be the root of the tree. And we define the parent-child relationship between each pair of GO terms with the basic GO relationships mentioned above. The parent-child relationship is defined as follows:

1. If $\mathrm{GO} 1$ "is a" $\mathrm{GO} 2$, we define $\mathrm{GO} 1$ as the parent of $\mathrm{GO} 2$

2. If GO1 is "part of" $\mathrm{GO} 2$, we define $\mathrm{GO} 2$ as the parent of $\mathrm{GO} 1$

3. If $\mathrm{GO} 1$ "regulates" $\mathrm{GO} 2$, we define $\mathrm{GO} 1$ as the parent of $\mathrm{GO} 2$

4. If GO1 "positively regulates" GO2, we define $\mathrm{GO} 1$ as the parent of GO2

5. If GO1 "negatively regulates" $\mathrm{GO} 2$, we define $\mathrm{GO} 1$ as the parent of GO2

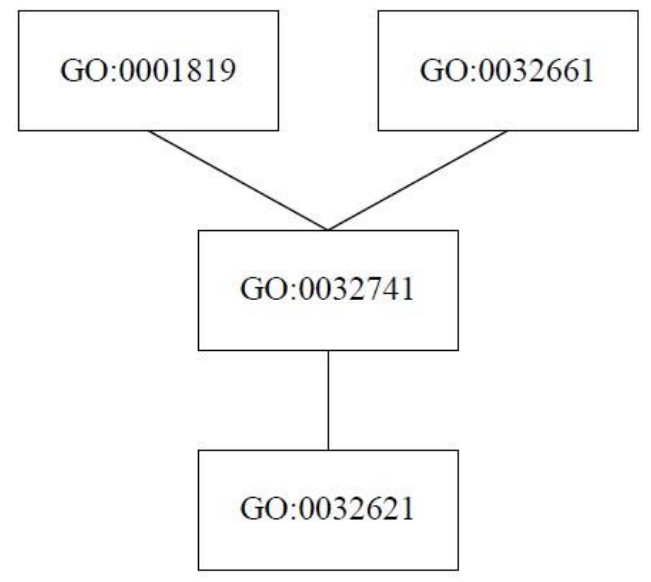

Figure 20: GO relation example

In Figure 3-2, we can see a part of tree represent the GO relations specified in Figure 20.

\subsection{Algorithm used to generate GO tree}


We design an algorithm to generate GO trees for biological process, cellular component and molecular function separately. Here we select the biological process as an example to introduce our algorithm:

Our algorithm contains two parts. Part 1figures out all the GO terms and their relations exist in the tree, i.e. vertices and edges exist in the tree. Part 2 figures out the layer information, i.e. the structure of the tree.

\section{Part 1:}

Step 1.Create hash tables for "is a" relation, "part of" relation, "regulates" relation, "positively regulates" relation and "negatively regulates" relation respectively. We hash all these five relationships of biological process GO terms exist in ontology file into corresponding hash tables. Keys represent the parent and values represent the children.

Step 2.Read the GO list, extract all the biological process GO terms and push them into an array - @GO_p.

Step 3.Each time we pop an element e from the head of @GO_p, check the all the basic relations of e, get a list of all the target GO terms. Scan the target GO list, and push those GO terms that did not appear before into the array @GO_p.

Step 4.Repeat step 3 until the @GO_p is empty. Then we get the integral list of GO relation pairs as well as the GO terms appear in the GO tree.

\section{Part 2:}

Step 1.Read the list of GO relation pairs, push all the GO terms with children in an array - @ parents, push all the GO terms with parents in another array @ children

Step 2.Determine the root of the whole tree. We know a GO term is the root iff it exists in array@parent but does not appear in the array@children.

Step 3.Push the root of the tree into a new created array - @ array. Create a hash \%hash_layer to store the layer of all GO terms. Initialize a variable \$current_layer which used to specify the current layer number to be 1 . The root is at layer 1 .

Step 4.Each time adds the value of \$current_layer by 1, pop out all the existing elements in @array, identify their children and store in array - @ array2. Scan @array2 and hash all the members in @array2 to \%hash_layer with value \$current_layer. Then override@array with@array2.

Step 5.Repeat step 4 until the @ array is empty. Now we gain all the layer information in the \%hash_layer. 


\subsection{Usage of online tool - GO tree}

There are two ways to plot the GO tree by using our online tool.

Method 1: Paste a list of a GO term and their p-values, make sure they are separated by a tab symbol. Then select the type of GO tree (biological process, cellular component or molecular function) you want to plot at the radiobox.

Click "submit" button.

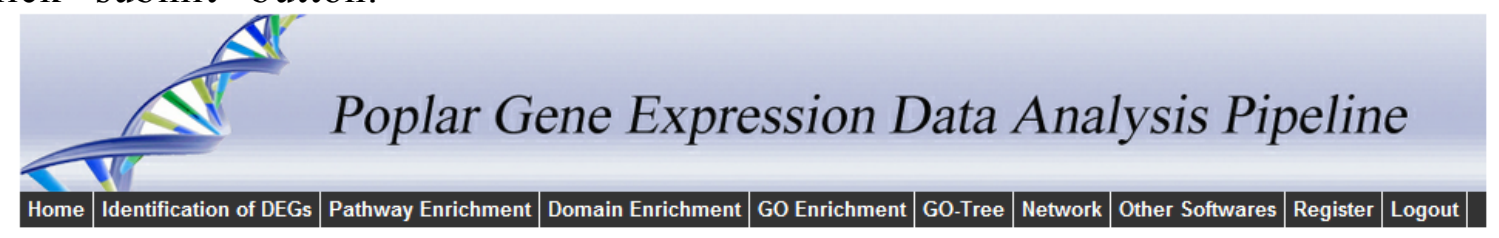

allen576! Welcome to use our GO-Tool ! Logout Click here!

Run GO-Tree Tool

Please input the list of GO Terms ( Example )

\begin{tabular}{|ll} 
GO: 0008152 & $9.35 \mathrm{E}-37$ \\
$\mathrm{GO}: 0055114$ & $6.24 \mathrm{E}-28$ \\
$\mathrm{GO}: 0046686$ & $9.58 \mathrm{E}-20$ \\
$\mathrm{GO}: 0006091$ & $9.93 \mathrm{E}-20$ \\
$\mathrm{GO}: 0005975$ & $2.37 \mathrm{E}-19$
\end{tabular}

Or upload the GO Terms file ( Example )

Choose File No file chosen

Please choose the tree you want to render Biological Process $\square$

Submit Reset

Figure 21: Paste the list of GO term along with their pvalues 


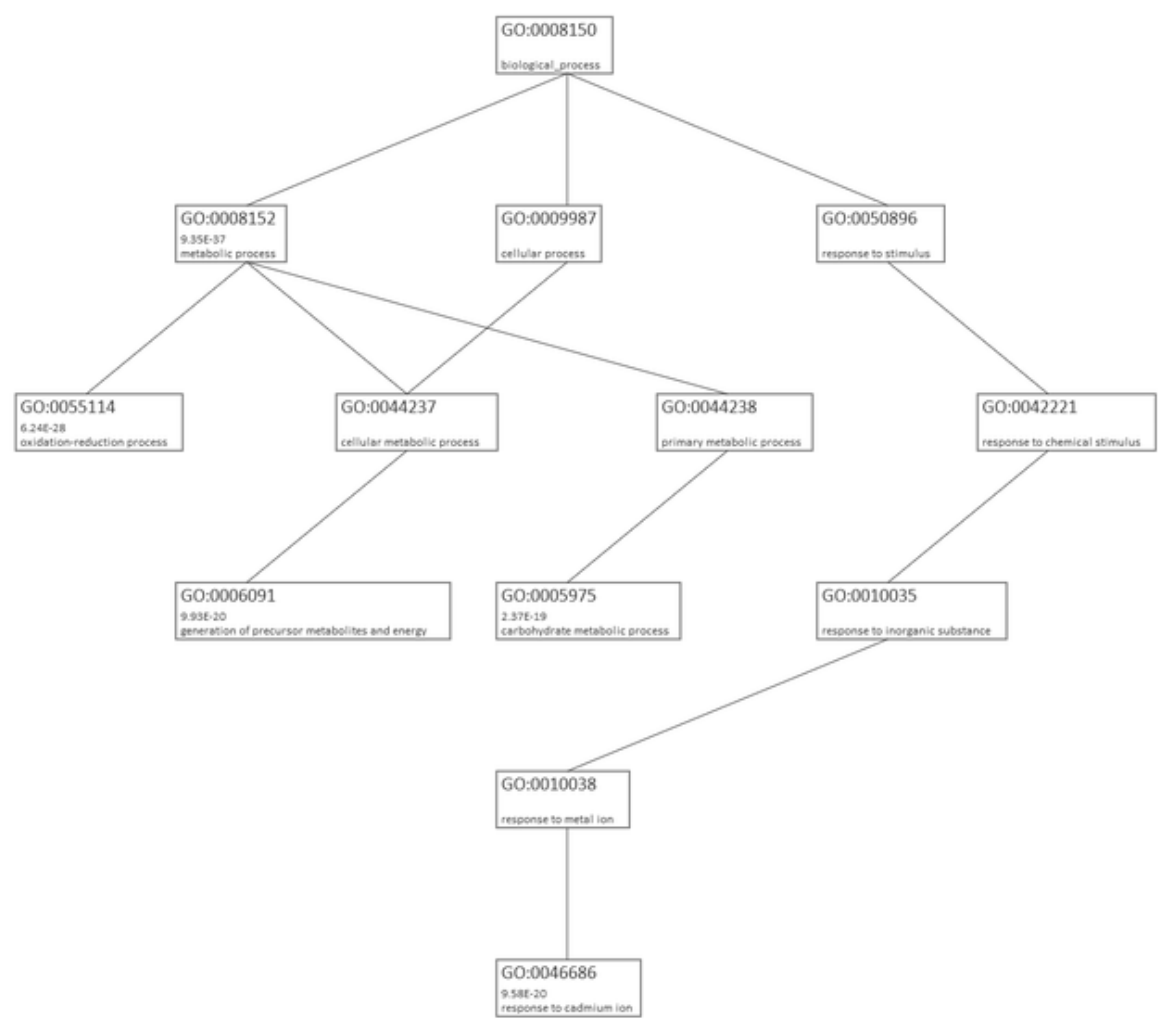

Figure 22: GO-Tree result (biological process)

Method 2: Upload a two column tab-delimited file that is resulting from you GO enrichment analysis. Column 1 contains GO terms, Column 2 provides the $p$ values of each GO term. The sample input file is specified in Figure 23. Then select the type of GO tree (biological process, cellular component or molecular function) one wants to plot at the radio box. Click "submit" button.

\begin{tabular}{|c|c|c|}
\hline & \multicolumn{1}{|c|}{ A } & B \\
\hline 1 & GO:0008152 & $9.35 \mathrm{E}-37$ \\
\hline 2 & GO:0055114 & $6.24 \mathrm{E}-28$ \\
\hline 3 & GO:0046686 & $9.58 \mathrm{E}-20$ \\
\hline 4 & GO:0006091 & $9.93 \mathrm{E}-20$ \\
\hline 5 & GO:0005975 & $2.37 \mathrm{E}-19$ \\
\hline
\end{tabular}

Figure 23: Upload GO-Tree file format 


\section{Chapter 4}

\section{GO Hierarchy Analysis Poplar Gene Expression Data Analysis On-line Tool}

\subsection{Introduction to poplar gene expression data analysis on-line tool}

Poplar Gene Expression Data Analysis Pipeline is an online tool designed for analyzing gene expression data from poplar. The URL is "http://sys.bio.mtu.edu". This tool is the web application of our pipeline program. It can analyze both microarray gene expressions and high-throughput gene expressions. For high-throughput gene expression, we only take the latest data version - version 3. Here is an introduction of our online tool.

\subsection{User Registration and Portion}

\subsubsection{Register}

Users must register before using web tool. The user registration page is shown in Figure 24: 


\section{Poplar Gene Expression Data Analysis Pipeline}

11

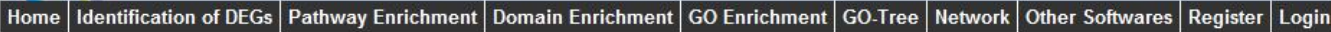

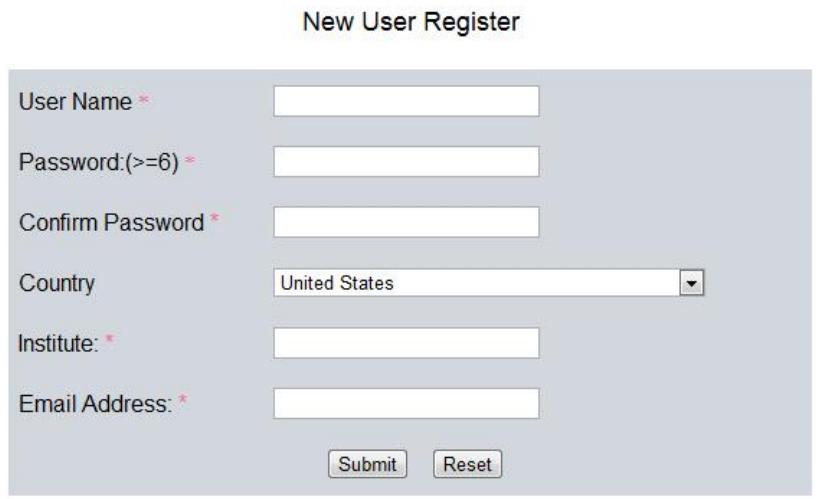

The fill marked * is required

Figure 24: User registration webpage

\subsubsection{Duplicate username}

We store the user registration information in our database. The user name must be unique. We will check the username in our database once a user submits a user ID. If we find this user name is already registered, the user will be directed to the webpage shown in Figure 25: 
Sorry! This name (allen576) has been taken.

Home | Services | Destinations | About Us | Contact Us

Copyright 2012 MTU School of Forest | Designed by Yufeng Guo and Xiang Li

Figure 25: Duplicate username error

\subsubsection{Duplicate Email}

The Email address also has to be unique. After completing the analyses requested, we will send the URL links of results to users via email. Our tool can automatically search the database to check if a given email address is already registered. If a given email has already been registered, users will be directed to the webpage shown in Figure 26: 


\section{Poplar Gene Expression Data Analysis Pipeline}

\begin{tabular}{|l|l|l|l|l|l|l|l|l|l} 
Home & Identification of DEGs & Pathway Enrichment & Domain Enrichment & Go Enrichment & GO-Tree & Network & Other Softwares & Register & Login \\
\hline
\end{tabular}

Welcome! Please login first! Click here!

Sorry! This email (xli5@mtu.edu) has been registed by other users

Home | Services | Destinations | About Us | Contact Us

Copyright 2012 MTU School of Forest I Designed by Yufeng Guo and Xiang Li

Figure 26: Duplicate email error

\subsection{User Login Portion}

\subsubsection{Login}

Each time users want to use the online tool, they have to login first. Users can either click the "Login" on the main menu or the hyperlink on up-right of each page, as shown in Figure 27:

\section{Welcome! Please login first! Click here!}

Figure 27: Login hyperlink

The login webpage is shown in Figure 28: 


\section{Please login first}

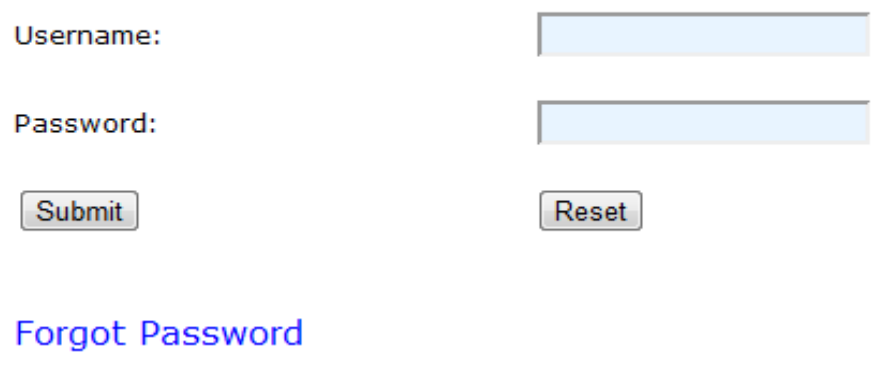

Figure 28: User login webpage

Make sure the username and password are correct. Otherwise an alert box will pop out, which is shown in Figure 29:

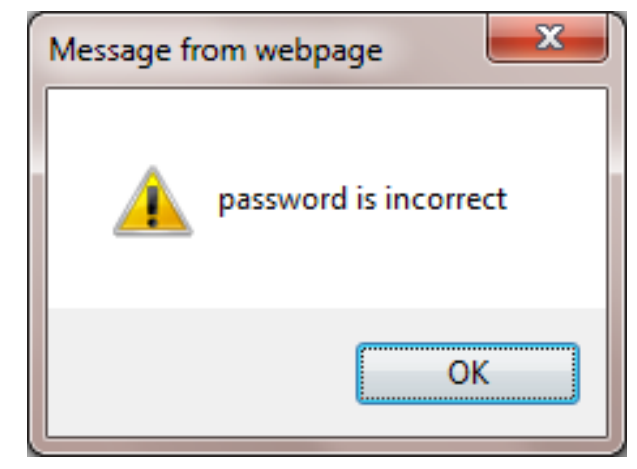

Figure 29: Alert box indicates the password is incorrect

\subsubsection{Password retrieval}

If users forgot their password, click the link - "Forgot Password", then website will redirect users to a find password webpage, it is shown in Figure 30:

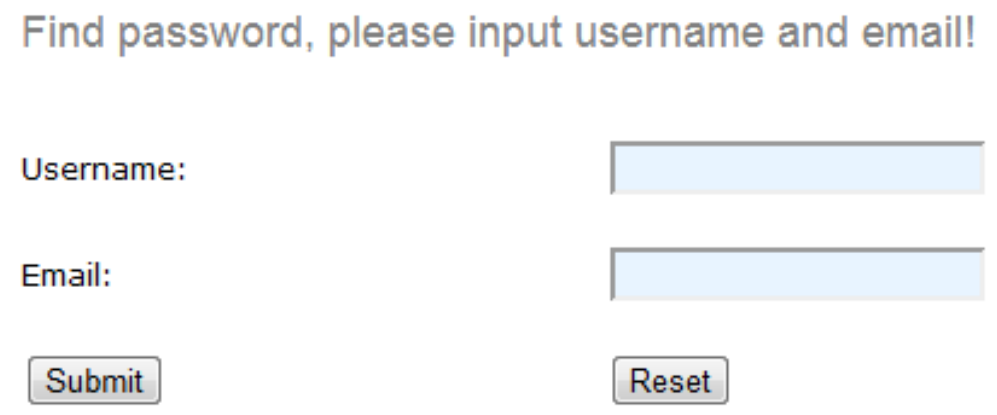

Figure 30: Finding password webpage

Users can retrieve their password by entering the usernames and registered email addresses in this text bar. Once the users click "Submit", our server will search 
the database immediately. If a match is found, the server extracts the password and sends it to the registered email address.

\subsection{Data analysis Pipeline}

After the user logins in, he/she can do the data analysis by using the online tool. All the analyses are specified in the main menu bar, which is shown in Figure 31:

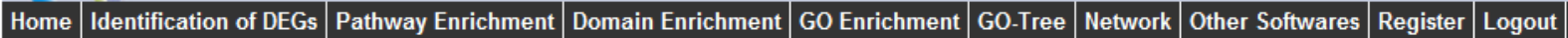

Figure 31: Menu Bar of pipeline Analysis

In Fig 4-8, we can see Identification of DEGs - DEG Analysis, Pathway Enrichment Analysis, Domain Enrichment Analysis, GO Enrichment Analysis and GO-Tree. These analyses all specified in pervious chapters. Here we only introduce the usage of online tool.

\subsubsection{Identification of DEGs}

In perform DEG analysis, users need to click "Identification of DEGs" in the main menu bar, the webpage is shown in Figure 32: 


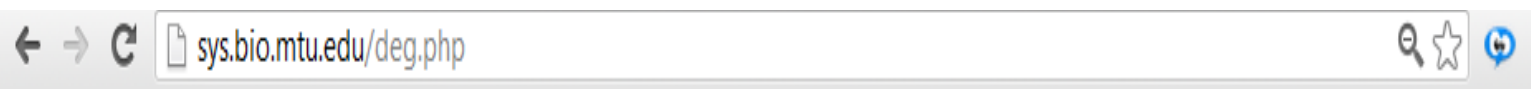

Identification of Differentially Expressed Genes(DEGs)

1. Select data platiorm type:

Miroogray $\quad$ -

2. Upload the gene expression data file: (Example: Micoarray data ) ( Example: High-throughput sequencing data )

Choose File No file chosen

Note that: (1) Controls column names must begin with $\mathrm{C}$ or $\mathrm{Q}$, whereas treatment column names begin with $\mathrm{T}$ or ti, (2) For each time

point comparison, control data are listed at left, and treatment data at right.

3. Enter the NUMBER of all time points or comparisons (e.g. treatments vs control):

2

4. Microarray Data (only): Precentage for trimming (lowly expressed genes) (e.g. 0.1'-10\%; 0 do not trim):

0.2

5. High Throughput Sequencing Data (only) : Enter the minimum values allowed.'(Set values smaller than this to this value. Default minimum value is 1)

1

5. Enter cut-off corrected p-value(FDR) for selecting DEGS (e.g. 0.05):

0.05

6. Do you want to run the following analyses upon running DEG analysis? (you are encouraged to run them at one time.

However, you can always examine the DEG output first and then come back to load the DEG output and run the following

analyses at a later time)

Metabolic Pathway Enrichment Analysis (Gene Set Enrichment Analysis)

$\square$ Protein Domain Enrichment Analysis

$\square$ Gene Ontology (GO) Enrichment Analysis

Next

Figure 32: DEG analysis webpage

In this webpage, users can specify the following parameters: 
1) Data type of the uploaded data, either "microarray" or "high-throughput".

2) Gene expression data file, click "Choose File" button and upload the gene expression data file from local PC. Make sure the format of uploaded file is correct. You can click the example link to check the sample file.

3) Time point number

4) For microarray data, specify the trimming percentage of the gene expression data.

5) For high throughput sequencing data, specify the minimum normalized value.

6) Cut-off threshold of corrected p-value. We will classify the poplar genes with corrected $p$-value smaller than this threshold as the DEGs

7) Analyses one wants to do after the DEG analysis in the checkboxes. There are three checkboxes - DEG sets Analysis (Pathway Analysis), Domain Analysis and GO Term Analysis. These checkboxes provide huge convenience in gene expression analysis. Users can choose all of them and then the online tool will do all the analyses once in the background. Once the analyses are completed, server will send all the results to the users using registered email addresses.

Click "next" and go to the confirmation page, as shown in Figure 33: 


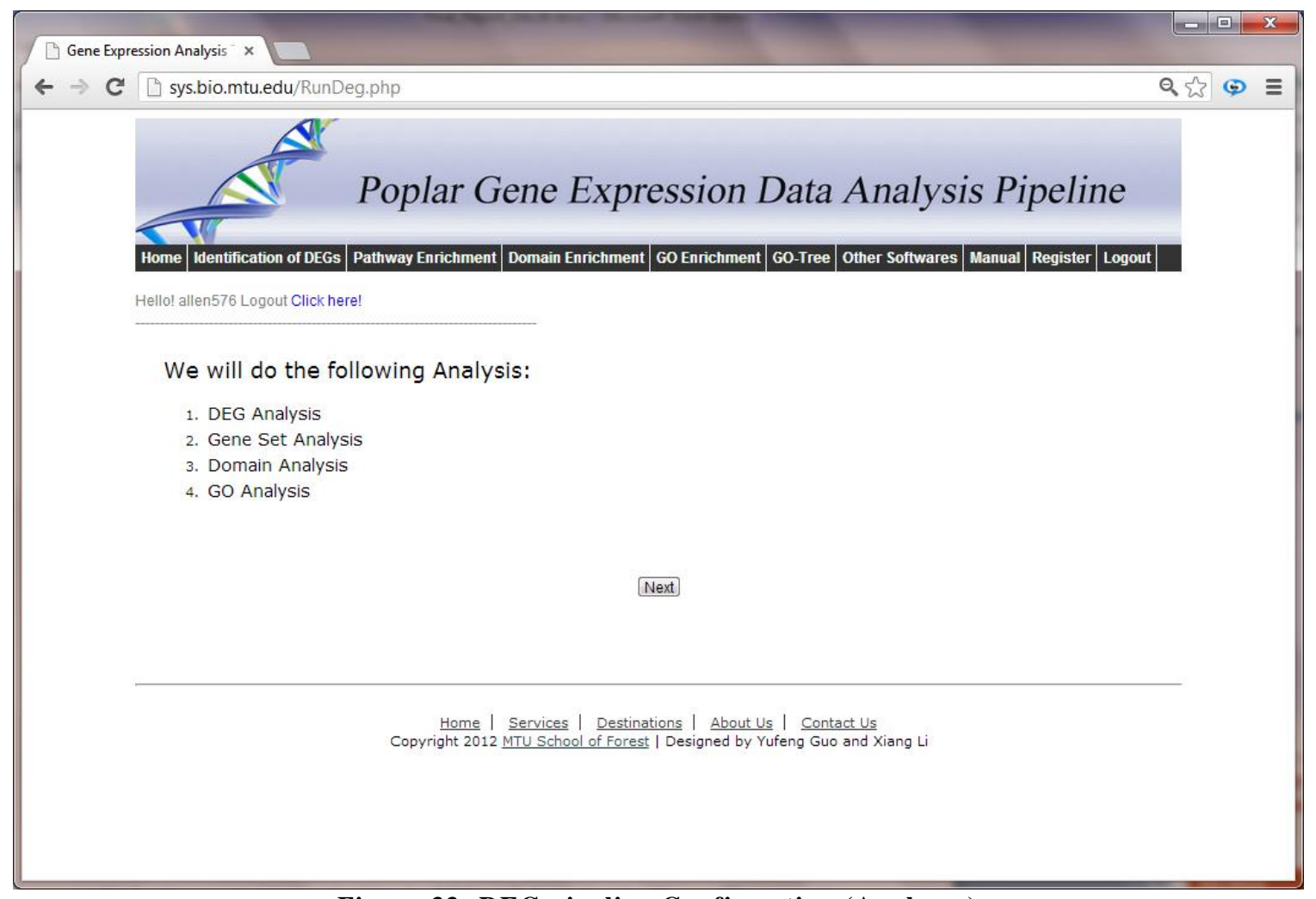

Figure 33: DEG pipeline Confirmation (Analyses)

Server will show all the analyses we will do in this page, if nothing is wrong, click "next" button. Then the users are redirected to web page that used to confirm the parameters, as shown in Figure 34: 


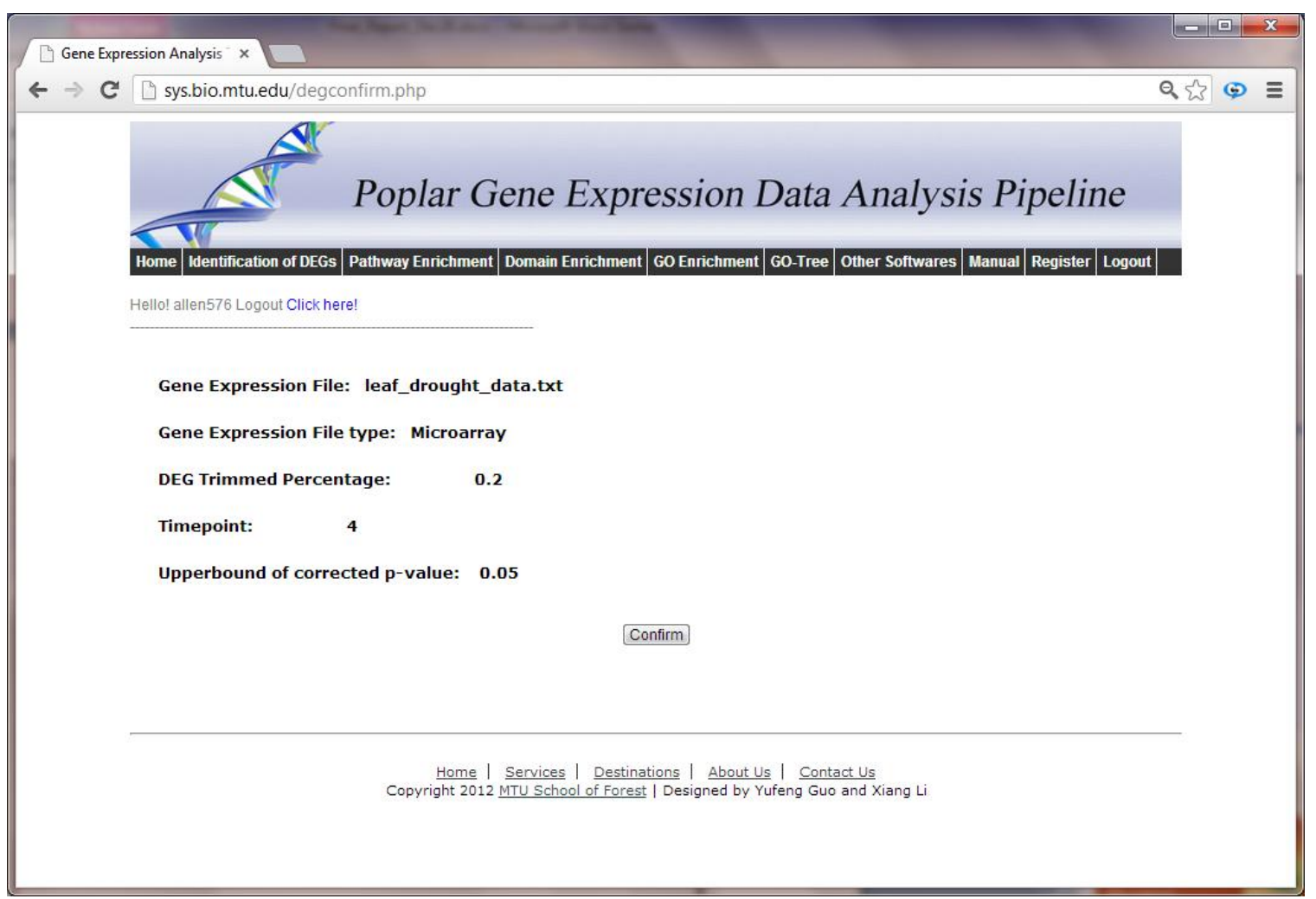

Figure 34: DEG pipeline Confirmation (parameters)

In the web page shown in Figure 34, one will find all the parameters he entered before. If all the parameters are correct, click "confirm" button. Then he will be redirected to the final webpage, as shown in Figure 35: 


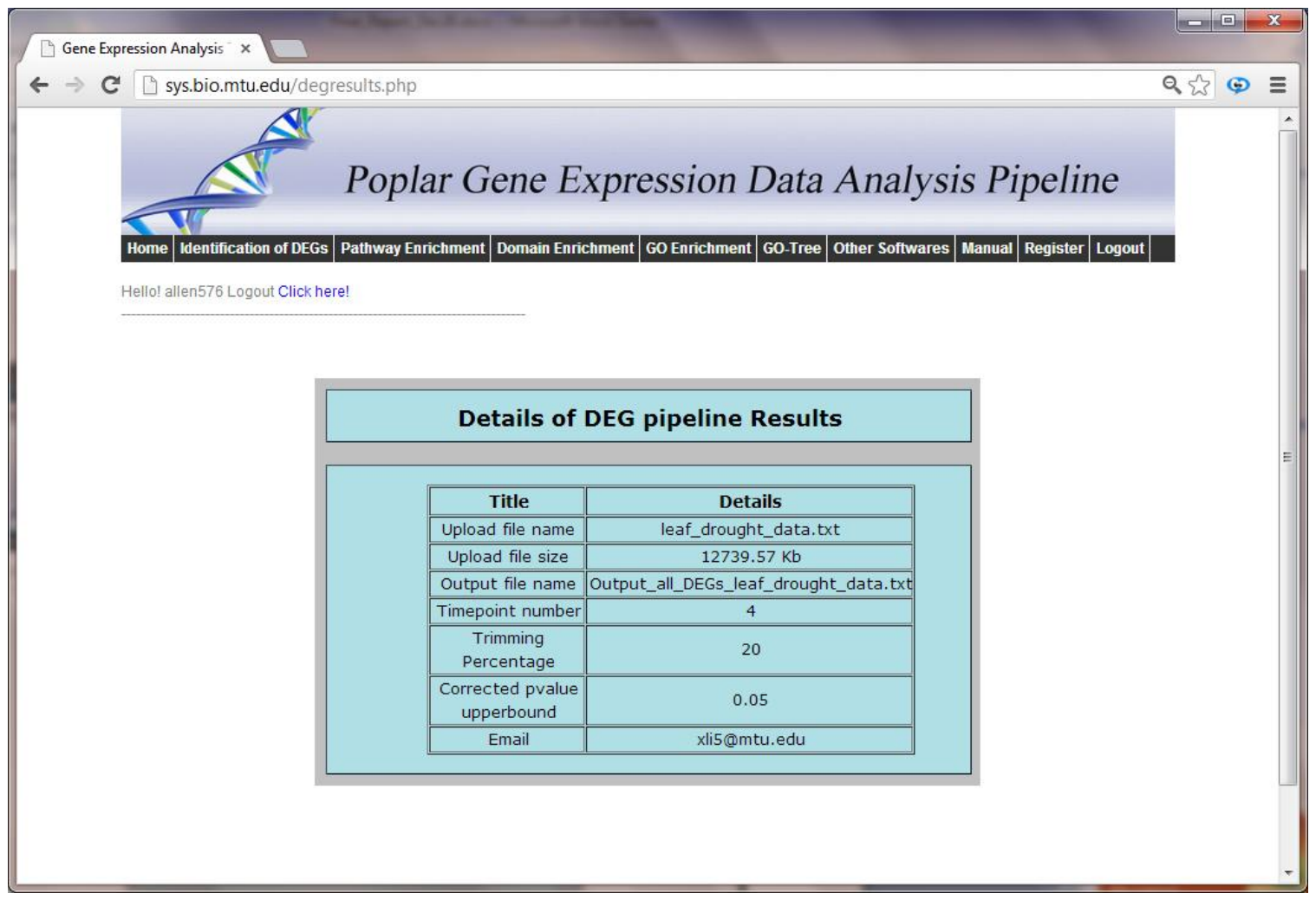

Figure 35: Final page of DEG pipeline

In Figure 34, users can get a form that specifies how the server will run the program. We can see the form contains the upload file name, upload file size, output file name, time point number, trimming percentage, upper bound value of corrected p-value which used to classify the DEGs, and the email address which the result will be sent to. Once users see this webpage, it indicates the tool has already got started to run the analysis. Users can logout and check their email after several hours.

Although user can do each pipeline analysis separately, we highly recommend users use this pipeline to do all the analyses at once. This provides some advantages in using the resource. Our online tool also checks the input files. Users will be directed to error warning webpage if they make mistakes of the file format. For example, Figure 36 shows the error warning webpage when user enters wrong time point number: 


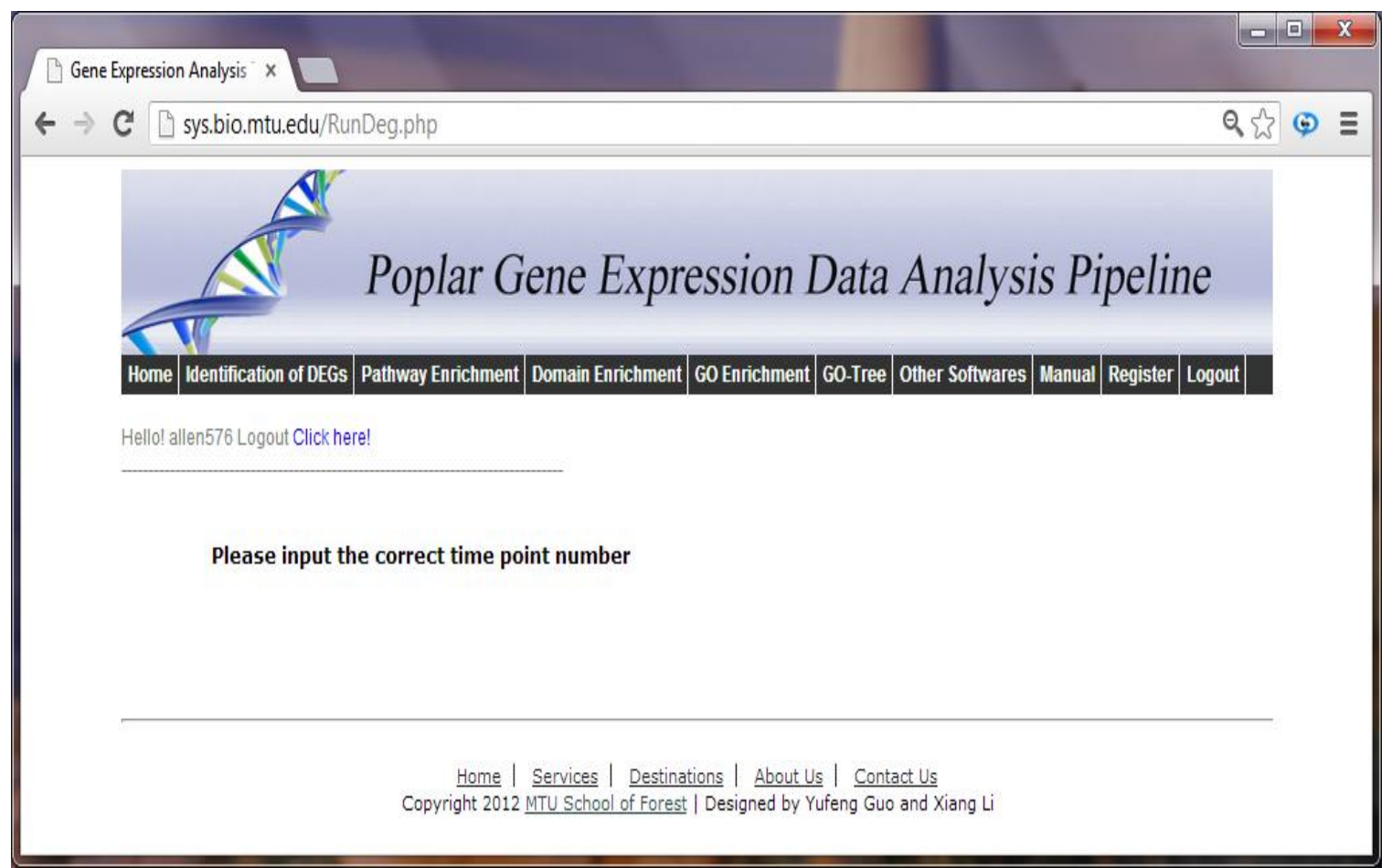

Figure 36: Wrong time point number warning

Our server uses this email-sysbiomtu@gmail.com. The output files will be sent to user's email address. A few example messages from our server are shown in Figure 37:

\section{Running Result $\quad \begin{array}{lll}\operatorname{Inb} b x & \mathrm{x} & \overline{1}\end{array}$}

\section{sysbiomtu@gmail.com 9:57 PM (1 hour ago)}

to me -

Microarray data DEG result:

http://sys.bio.mtu.edu/user/allen576/running_result/

output_all_DEGs_leaf_drought_data.txt

\section{sysbiomtu@gmail.com}

to me -

Microarray data DEG set analysis results:

http://sys.bio.mtu.edu/user/allen576/running_result/

Enriched_pathways_all_leaf_drought_data.txt

Figure 37: Results get from server 
Users can download the results by clicking the links in the email.

\subsubsection{Pathway Enrichment Analysis}

If one wants to do the pathway enrichment analysis, he needs to click the "Pathway Enrichment" in the main menu bar.

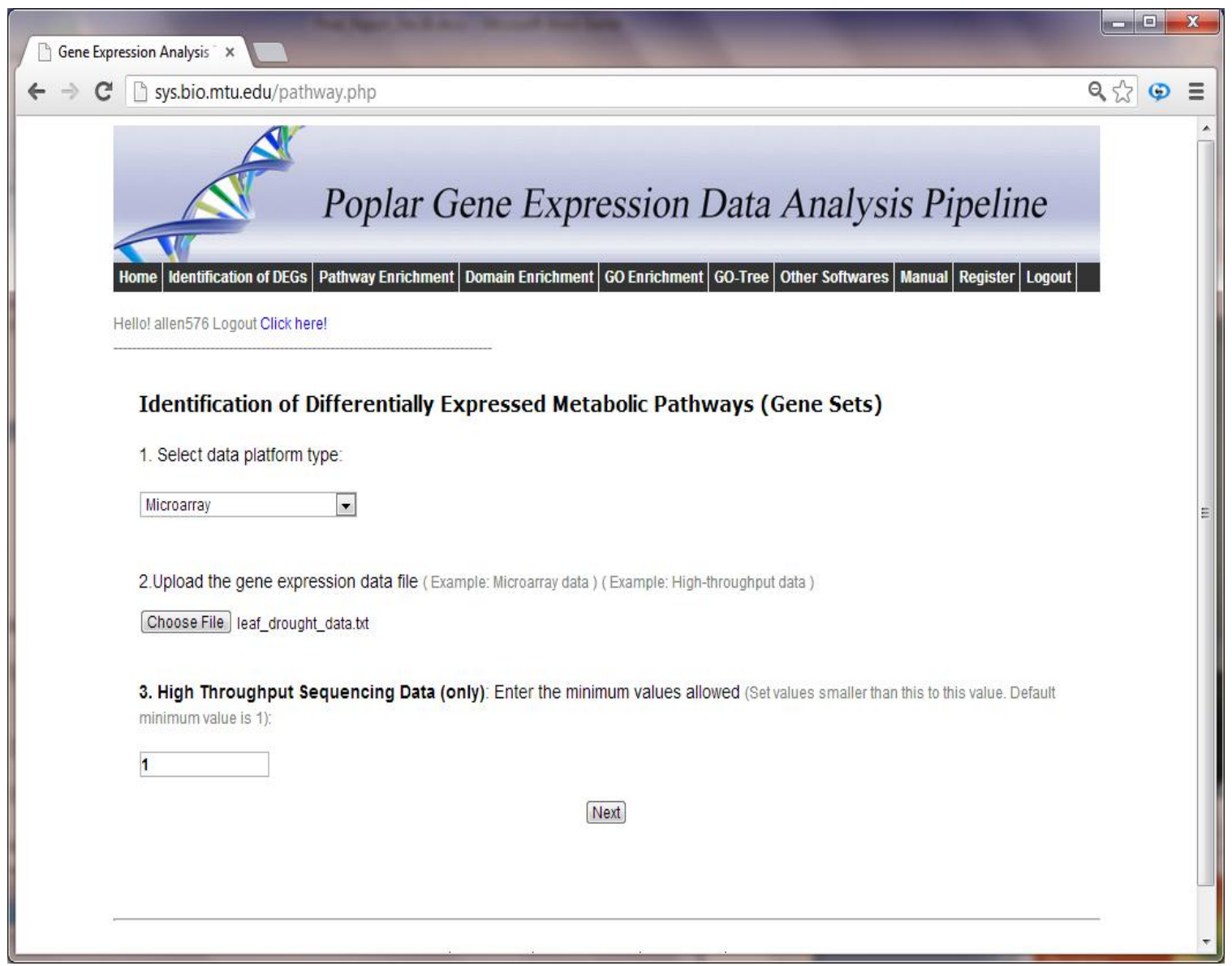

Figure 38: Pathway Enrichment Analysis

In this webpage, users can:

1) Choose the data type of the uploaded data, either "microarray" or "highthroughput".

2) Click "Browse" button and upload the gene expression data file from local PC.

3) For high throughput sequencing data, enter the minimum normalized value. 
After entering all the parameters, click "next". We will see the confirmation webpage shown in Figure 39:

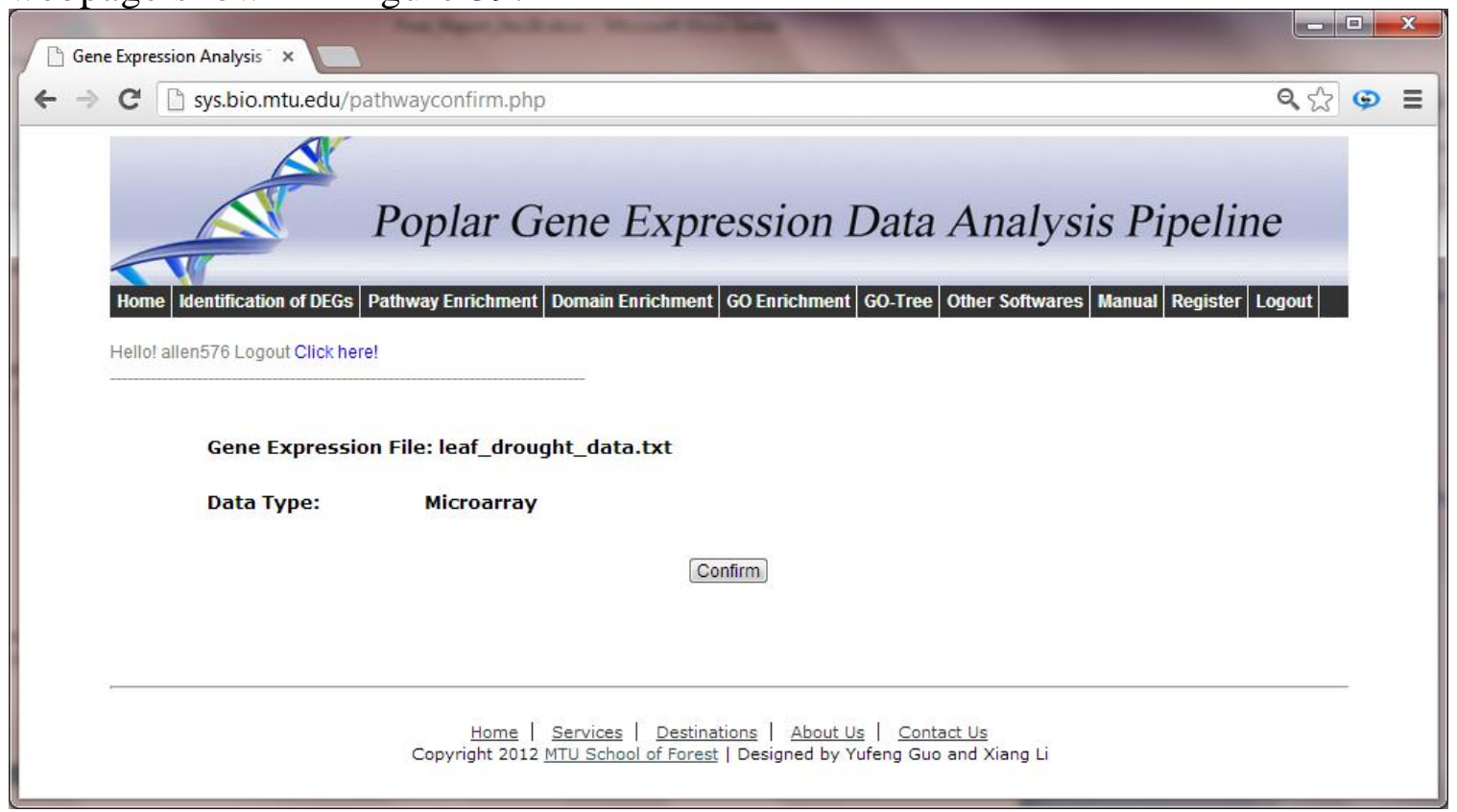

Figure 39: Confirmation page of pathway enrichment analysis

After confirming the parameters entered, click "confirm" button and make the server run the program. The final webpage of pathway enrichment analysis is shown in Figure 40.

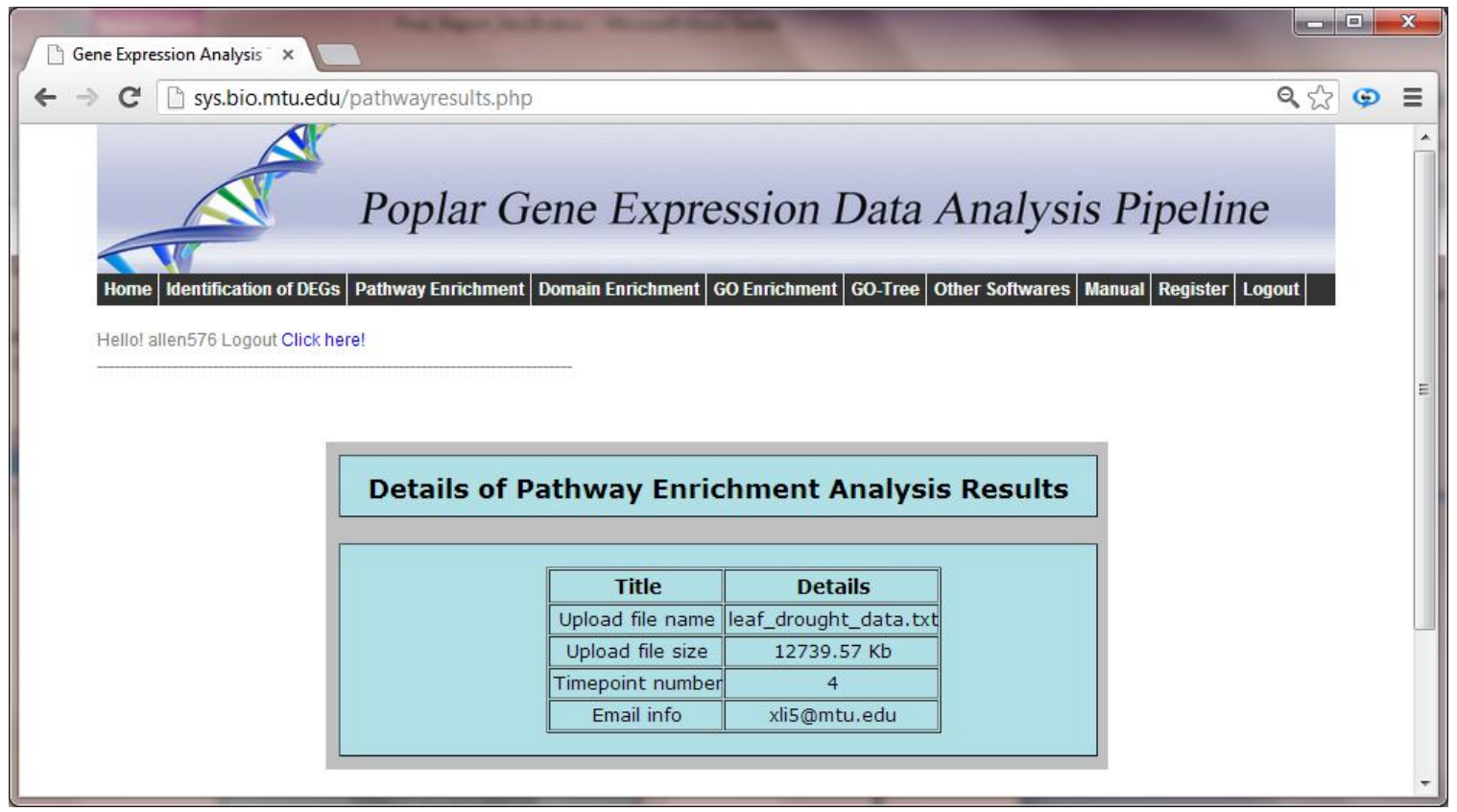

Figure 40: Final result of pathway enrichment 
Webpage in Figure 40 shows the details of upload file, time point number and the email address that the server will send the outputs to.

For the pathway enrichment analysis, we currently do not allow user to upload their pathway matrix file. Our server has its owns pathway matrix file for poplar. It is composed of 340 pathways.

\subsubsection{Domain Enrichment Analysis}

If one wants to identify enriched domains in a list of DEGs, first clicks the "Domain Enrichment" in the main menu bar.

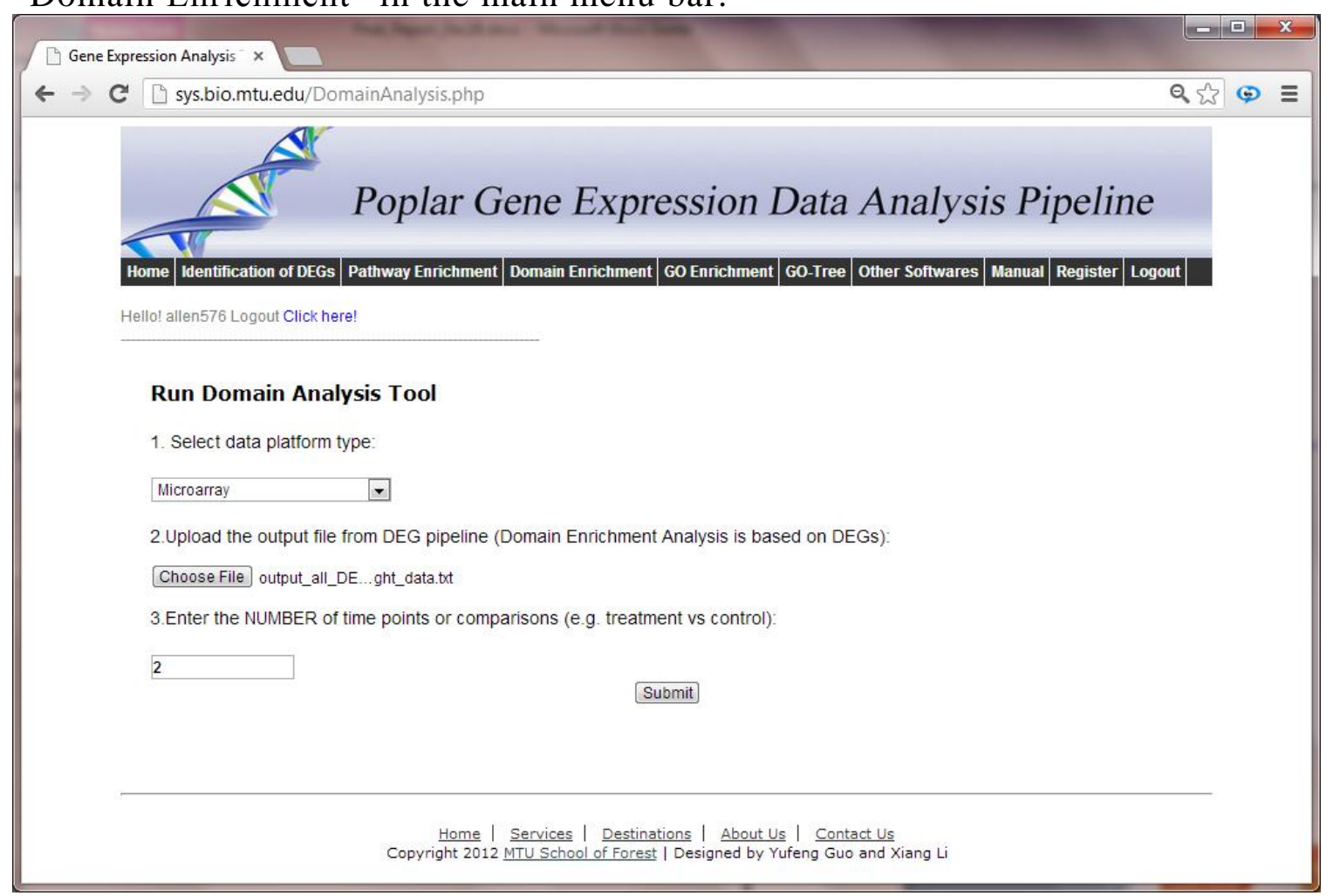

Figure 41: Domain Enrichment Analysis

From Figure 41 we can see users need to specify the following parameters to run the domain enrichment analysis:

1) Choose the data type of the uploaded data, either "microarray" or "highthroughput".

2) Click "Choose Files" button and upload the output file of DEG pipeline.

Similar with pathway enrichment analysis, users will be directed to the confirmation page that contains the upload file name and time point number. 


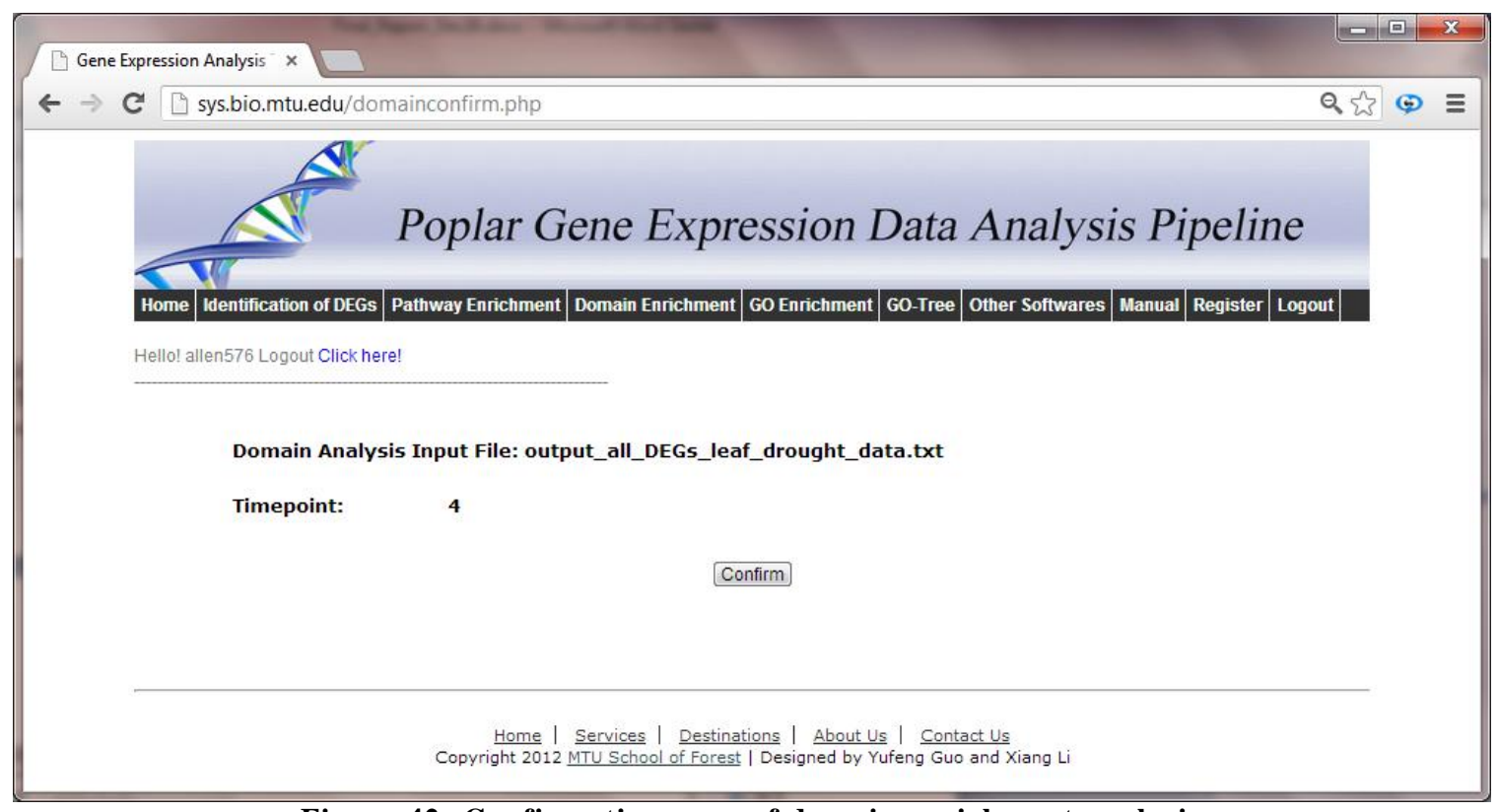

Figure 42: Confirmation page of domain enrichment analysis

After confirming the input file and time point number, click "confirm" button to run the program. The final webpage of pathway enrichment analysis is shown in Figure 43.

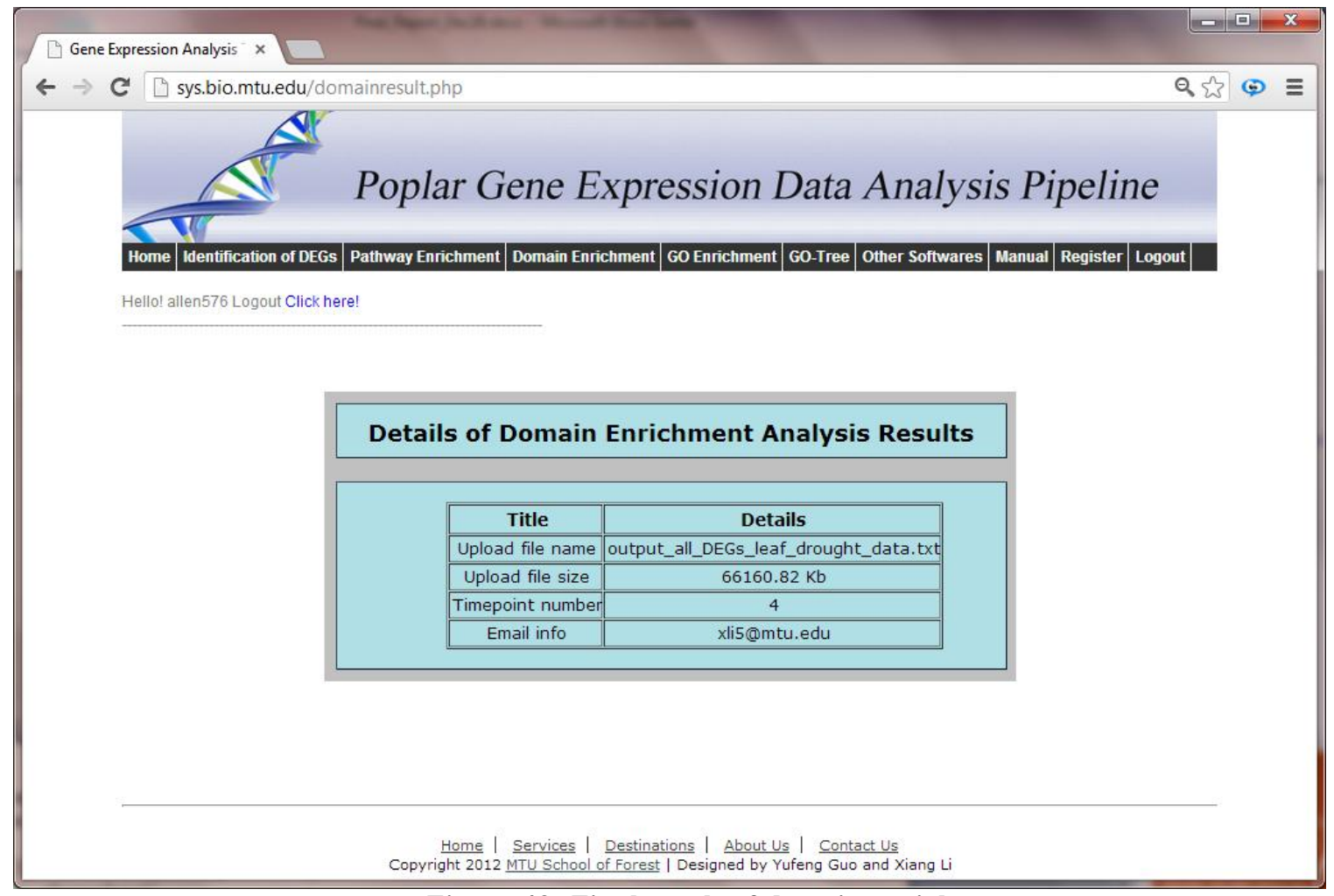

Figure 43: Final result of domain enrichment 
For domain enrichment analysis, our tool already has the protein domain annotation file in place. Users only need to upload the protein domain annotation file. We also keep track of the latest domain annotation file and renew the annotation file to produce the results up to date.

\subsubsection{GO Enrichment Analysis}

If one wants to do the GO enrichment analysis, first click the "GO Enrichment" in the main menu bar.

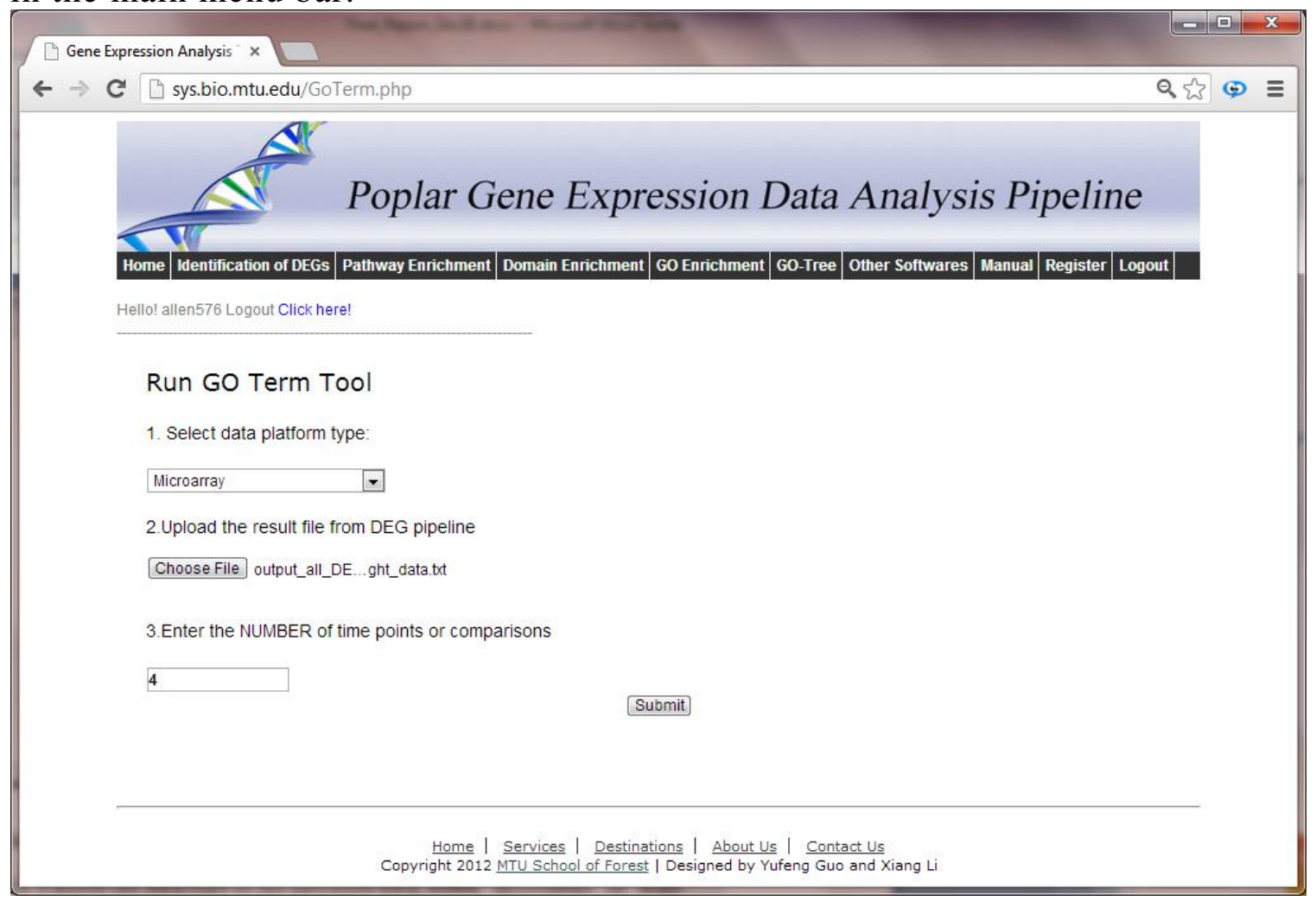

Figure 44: GO Enrichment Analysis

In this webpage, users have to:

1) Choose the data type of the uploaded data, either "microarray" or "highthroughput".

2) Click "Choose Files" button and upload the output file from DEG pipeline.

3) Enter the number of time points in the text box.

Similar with pathway enrichment analysis and domain enrichment analysis, users are directed to the confirmation page that contains the upload file name and time point number. 


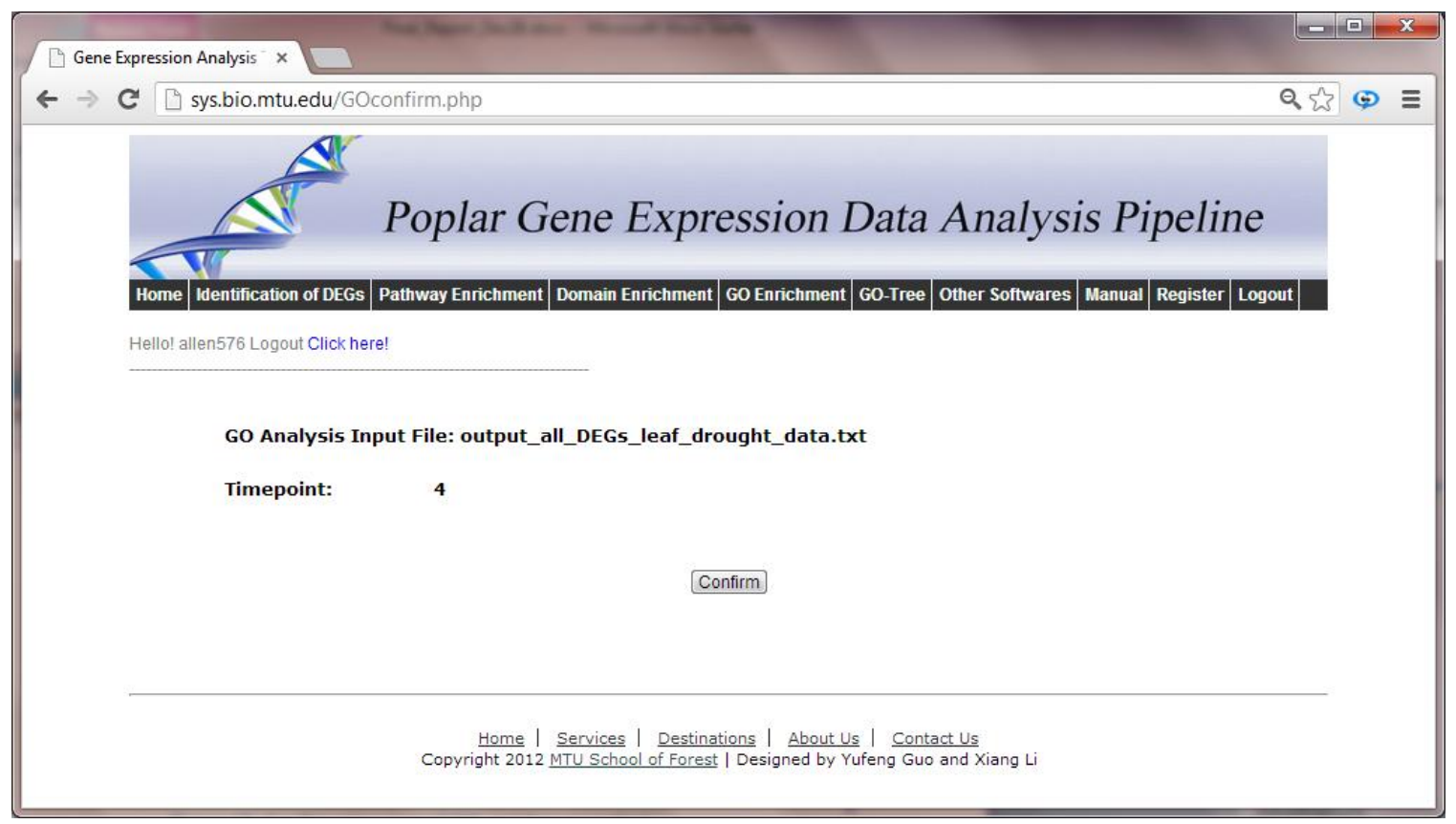

Figure 45: Confirmation page of GO enrichment analysis

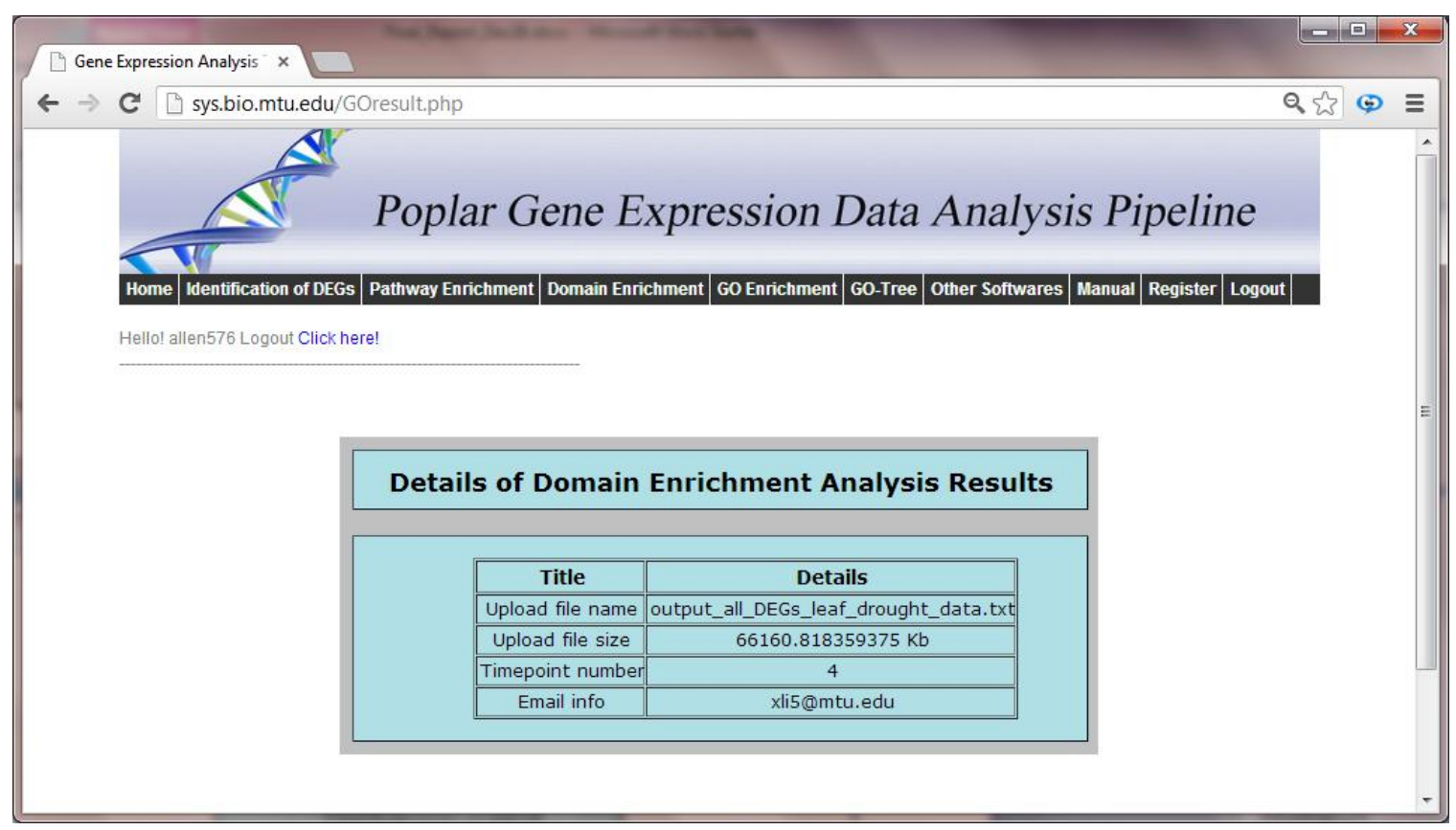

Figure 46: Final result of GO enrichment

For the GO enrichment analysis, users only need to upload the output file resulting from DEG pipeline. Our tool already has the GO ontology file and annotation files, Arabidopsis genes' annotation file and GO annotation file. We 
keep track of the latest lease of annotation files and GO annotation file and update of files once the new annotation become available. 


\section{Conclusion and Future Work}

The purpose of this project is to develop a set of tools for rapid analyzing gene expression data for poplar genes. The pipelines can be used to identify of DEGs, enriched pathways, enriched domains and enriched GO terms. We also developed an on-line tool to enable users do the analyses of their data easily. Such tool can be used to analyze both microarray data and high throughput data.

Some potential contents that can be integrated to improve our pipeline tool are as follows:

1. Integrate multiple methods to identify the DEGs, add an option to the methods so that users can choose proper methods for their data.

2. Add more automated visualization schemes, for example heat-map or bar-plot to automatically show the average fold change or other information we are interested in.

3. Add more species, currently our tool can only analyze poplar gene expression data.

4. Add methods to build gene network and automatically identify regulatory modules

5. Add time execution time bar to let users know how much time it needs to get the running results. 


\section{Reference:}

1. Wolfgang Huber. "Analysis of microarray gene expression data"

2. Paul J.Hurd and Christopher J.Nelson " Advantages of next-generation sequencing versus the microarray in epigenetic research"

3. Breitling, R., Armengaud, P., Amtmann, A., and Herzyk, P.(2004) "Rank products: a simple, yet powerful, new method to detect differentially regulated genes in replicated microarray experiments."

4. Hall N (May 2007). "Advanced sequencing technologies and their wider impact in microbiology". J. Exp. Biol. 210 (Pt 9): 151825.

5. Church GM (January 2006). "Genomes for all". Sci. Am. 294 (1): 46-54.

6. Kadota, K., Y. Nakai, and K. Shimizu, A weighted average difference method for detecting differentially expressed genes from microarray data. Algorithms Mol Biol, 2008.3: p. 8.

7. Smyth, G.K., Linear models and empirical bayes methods for assessing differential expression in microarray experiments. Stat Appl Genet Mol Biol, 2004. 3: p. Article3.

8. Benjamini, Yoav; Hochberg, Yosef (1995). "Controlling the false discovery rate: a practical and powerful approach to multiple testing". Journal of the Royal Statistical Society, Series B (Methodological) 57 (1): 289-300.

9. Genetics, S., Multiple Testing Corrections. 2003.

10. Irina Dinu, John D Potter, Thomas Mueller, Qi Liu, Adeniyi J Adewale, Gian S Jhangri, Gunilla Einecke, Konrad S Famulski, Philip Halloran and Yutaka Yasui (2007) "Improving gene set analysis of microarray data by SAM-GS"

11. Mootha VK, Lindgren CM, Eriksson KF, Subramanian A, Sihag S, Lehar J, Puigserver P, Carlsson E, Ridderstrale M, Laurila E, et al.: "PGCL appharesponsive genes involved in oxidative phosphorylation are coordinately downregulated in human diabetes" Nat Genet 2003,34:267-273

12. The Gene Ontology Consortium (January 2008). "The Gene Ontology project in 2008". Nucleic Acids Res. 36 (Database issue)

13. Elizebeth I.Boyle, Shuai Weng, Jere Gollub, Heng Jin, David Botstein, J.Michael Cherry, Gavin Sherlock, "GO::TermFinder-open source software for accessing Gene Ontology information and finding significantly enriched Gene Ontology terms associated with a list of genes" Bioinformatics. 2004 Dec 12;20(18):3710-5. Epub 2004 Aug 5. 